\title{
Canonical bases of higher-level $q$-deformed Fock spaces
}

\author{
Xavier Yvonne
}

Received: 12 June 2006 / Accepted: 29 January 2007 /

Published online: 17 April 2007

(C) Springer Science+Business Media, LLC 2007

\begin{abstract}
We show that the transition matrices between the standard and the canonical bases of infinitely many weight subspaces of the higher-level $q$-deformed Fock spaces are equal.
\end{abstract}

\section{Introduction}

The $q$-deformed higher-level Fock spaces were introduced in [6] in order to compute the crystal graph of any irreducible integrable representation of level $l \geq 1$ of $U_{q}\left(\widehat{\mathfrak{s l}}_{n}\right)$. More precisely, the Fock representation $\mathbf{F}_{q}\left[s_{l}\right]$ depends on a parameter $s_{l}=\left(s_{1}, \ldots, s_{l}\right) \in \mathbb{Z}^{l}$ called multi-charge. It contains as a submodule the irreducible integrable $U_{q}\left(\widehat{\mathfrak{s l}}_{n}\right)$-module with highest weight $\Lambda_{s_{1}}+\cdots+\Lambda_{s_{l}}$. The representation $\mathbf{F}_{q}\left[s_{l}\right]$ is a generalization of the level-one Fock representation of $U_{q}\left(\widehat{\mathfrak{s l}}_{n}\right)([4,17]$, see also $[14,15])$.

The canonical bases are bases of the Fock representations that are invariant under a certain involution - of $U_{q}\left(\widehat{\mathfrak{s l}}_{n}\right)$ and that give at $q=0$ and $q=\infty$ the crystal bases. They were constructed for $l=1$ in [14, 15] and for $l \geq 1$ by Uglov [19]. In [19], Uglov provides an algorithm for computing these canonical bases. He also gives an expression of the transition matrices between the standard and the canonical bases in terms of Kazhdan-Lusztig polynomials for affine Hecke algebras of type $A$.

In this article we prove three theorems.

1. The first one (Theorem 3.9) is a generalization to $l \geq 1$ of a result of [13]. It compares the transition matrices of the canonical bases of some weight subspaces inside a given Fock space $\mathbf{F}_{q}\left[s_{l}\right]$. The weights involved are conjugated under the action of the Weyl group of $U_{q}\left(\widehat{\mathfrak{s l}}_{n}\right)$. This action leads to bijections $\sigma_{i}$ that can

\footnotetext{
X. Yvonne $(\bowtie)$ Institut Camille Jordan (Mathématiques), Université Lyon I, 43 Bd du 11 Novembre 1918, 69622 Villeurbanne Cedex, France e-mail: yvonne@math.univ-lyon1.fr
} 
be described in a combinatorial way by adding/removing as many $i$-nodes as possible to the $l$-multi-partitions indexing the canonical bases. These bijections are generalizations of the Scopes bijections introduced in [18] in order to study, when $n=p$ is a prime number, the $p$-blocks of symmetric groups of a given defect.

2. In a dual manner, our second result (Theorem 4.4) gives some sufficient conditions on multi-charges $s_{l}$ and $\boldsymbol{t}_{l}$ with given residues modulo $n$ that ensure that the transition matrices of the canonical bases of some weight subspaces of $\mathbf{F}_{q}\left[\boldsymbol{s}_{l}\right]$ and $\mathbf{F}_{q}\left[\boldsymbol{t}_{l}\right]$ coincide.

3. Our third result (Theorem 5.2) is an application of Theorem 4.4 to the case when the multi-charges $\boldsymbol{s}_{l}=\left(s_{1}, \ldots, s_{l}\right)$ and $\boldsymbol{t}_{l}=\left(t_{1}, \ldots, t_{l}\right)$ are dominant, that is $s_{1} \gg$ $\cdots \gg s_{l}$ and $t_{1} \gg \cdots \gg t_{l}$. It shows that the transition matrices of the canonical bases of the Fock spaces $\mathbf{F}_{q}\left[s_{l}\right]$ stabilize when $\boldsymbol{s}_{l}$ becomes dominant (with a given sequence of residues modulo $n$ ). This supports the following conjecture (see [22]). We conjecture that if $s_{l}=\left(s_{1}, \ldots, s_{l}\right)$ is dominant, then the transition matrix of the homogeneous component of degree $m$ of the canonical basis of the Fock space $\mathbf{F}_{q}\left[s_{l}\right]$ is equal to the decomposition matrix of the cyclotomic $v$-Schur algebra $\mathcal{S}_{\mathbb{C}, m}\left(\zeta ; \zeta^{S_{1}}, \ldots, \zeta^{s_{l}}\right)$ of [2], where $\zeta$ is a complex primitive $n$-th root of unity. This conjecture generalizes both Ariki's theorem for Ariki-Koike algebras (see [1]) and a result of Varagnolo and Vasserot (see [20]) which relates the canonical basis of the level-one Fock space and the decomposition matrix of $v$-Schur algebras with parameter a complex $n$-th root of unity.

Notation Let $\mathbb{N}$ (respectively $\mathbb{N}^{*}$ ) denote the set of nonnegative (respectively positive) integers, and for $a, b \in \mathbb{R}$ denote by $\llbracket a ; b \rrbracket$ the discrete interval $[a, b] \cap \mathbb{Z}$. For $X \subset \mathbb{R}, t \in \mathbb{R}, N \in \mathbb{N}^{*}$, put

$$
X^{N}(t):=\left\{\left(s_{1}, \ldots, s_{N}\right) \in X^{N} \mid s_{1}+\cdots+s_{N}=t\right\} .
$$

Throughout this article, we fix 3 integers $n, l \geq 1$ and $s \in \mathbb{Z}$. Let $\Pi$ denote the set of all integer partitions, and for $N \in \mathbb{N}^{*}$, let $\Pi^{N}$ denote the set of all $N$-multi-partitions. The empty partition (respectively empty $N$-multi-partition) will be denoted by $\emptyset$ (respectively $\emptyset_{N}$ ).

\section{Higher-level $q$-deformed Fock spaces}

In this section, we introduce the higher-level Fock spaces and their canonical bases. We follow here [19], to which we refer the reader for more details. All definitions and results given here are due to Uglov.

\subsection{The quantum algebras $U_{q}\left(\widehat{\mathfrak{s l}}_{n}\right)$ and $U_{p}\left(\widehat{\mathfrak{s l}}_{l}\right)$}

In this section, we assume that $n \geq 2$ and $l \geq 2$. Let $\widehat{\mathfrak{s l}}_{n}$ be the Kac-Moody algebra of type $A_{n-1}^{(1)}$ defined over the field $\mathbb{Q}$ [7]. Let $\mathfrak{h}^{*}$ be the dual of the Cartan subalgebra of $\widehat{\mathfrak{s l}}_{n}$. Let $\Lambda_{0}, \ldots, \Lambda_{n-1} \in \mathfrak{h}^{*}$ be the fundamental weights, $\alpha_{0}, \ldots, \alpha_{n-1} \in \mathfrak{h}^{*}$ be the simple roots and $\delta:=\alpha_{0}+\cdots+\alpha_{n-1}$ be the null root. It will be convenient to extend 
the index set of the fundamental weights by setting $\Lambda_{i}:=\Lambda_{i} \bmod n$ for all $i \in \mathbb{Z}$. The simple roots are related to the fundamental weights by

$$
\alpha_{i}=2 \Lambda_{i}-\Lambda_{i-1}-\Lambda_{i+1}+\delta_{i, 0} \delta \quad(0 \leq i \leq n-1) .
$$

For $0 \leq i, j \leq n-1$, let $a_{i, j}$ be the coefficient of $\Lambda_{j}$ in $\alpha_{i}$. The space

$$
\mathfrak{h}^{*}=\bigoplus_{i=0}^{n-1} \mathbb{Q} \Lambda_{i} \oplus \mathbb{Q} \delta=\bigoplus_{i=0}^{n-1} \mathbb{Q} \alpha_{i} \oplus \mathbb{Q} \Lambda_{0}
$$

is equipped with a non-degenerate bilinear symmetric form $(.$, .) defined by

$$
\left(\alpha_{i}, \alpha_{j}\right)=a_{i, j}, \quad\left(\Lambda_{0}, \alpha_{i}\right)=\delta_{i, 0}, \quad\left(\Lambda_{0}, \Lambda_{0}\right)=0 \quad(0 \leq i, j \leq n-1) .
$$

Let $U_{q}\left(\widehat{\mathfrak{s l}}_{n}\right)$ be the $q$-deformed universal enveloping algebra of $\widehat{\mathfrak{s l}}_{n}$. This is an algebra over $\mathbb{Q}(q)$ with generators $e_{i}, f_{i}, t_{i}^{ \pm 1}(0 \leq i \leq n-1)$ and $\partial$. Let $U_{q}^{\prime}\left(\widehat{\mathfrak{s l}}_{n}\right)$ be the subalgebra of $U_{q}\left(\widehat{\mathfrak{s l}}_{n}\right)$ generated by $e_{i}, f_{i}, t_{i}^{ \pm 1}(0 \leq i \leq n-1)$. The relations in $U_{q}^{\prime}\left(\widehat{\mathfrak{s l}}_{n}\right)$ are standard and will be omitted (see e.g. [10]). The relations among the degree generator $\partial$ and the generators of $U_{q}^{\prime}\left(\widehat{\mathfrak{s l}}_{n}\right)$ can be found in $[19, \S 2.1]$. If $M$ is a $U_{q}\left(\widehat{\mathfrak{s l}}_{n}\right)$-module, denote by $\mathcal{P}(M)$ the set of weights of $M$ and let $M\langle w\rangle$ denote the subspace of $M$ of weight $w$. If $x \in M\langle w\rangle \backslash\{0\}$ is a weight vector, denote by

$$
\operatorname{wt}(x):=w
$$

the weight of $x$. The Weyl group of $\widehat{\mathfrak{s l}}_{n}\left(\right.$ or $\left.U_{q}\left(\widehat{\mathfrak{s l}}_{n}\right)\right)$, denoted by $W_{n}$, is the subgroup of $\operatorname{GL}\left(\mathfrak{h}^{*}\right)$ generated by the simple reflections $\sigma_{i}$ defined by

$$
\sigma_{i}(\Lambda)=\Lambda-\left(\Lambda, \alpha_{i}\right) \alpha_{i} \quad\left(\Lambda \in \mathfrak{h}^{*}, 0 \leq i \leq n-1\right)
$$

Note that $W_{n}$ is isomorphic to $\widetilde{\mathfrak{S}}_{n}$, the affine symmetric group which is a Coxeter group of type $A_{n-1}^{(1)}$.

We also introduce the algebra $U_{p}\left(\widehat{\mathfrak{s l}}_{l}\right)$ with

$$
p:=-q^{-1} \text {. }
$$

In order to distinguish the elements related to $U_{q}\left(\widehat{\mathfrak{s l}}_{n}\right)$ from those related to $U_{p}\left(\widehat{\mathfrak{s l}}_{l}\right)$, we put dots over the latter. For example, $\dot{e}_{i}, \dot{f}_{i}, \dot{t}_{i}^{ \pm 1}(0 \leq i \leq l-1)$ and $\dot{\partial}$ are the generators of $U_{p}\left(\widehat{\mathfrak{s l}}_{l}\right), \dot{\alpha}_{i}(0 \leq i \leq l-1)$ are the simple roots for $U_{p}\left(\widehat{\mathfrak{s l}}_{l}\right), \dot{W}_{l}=$ $\left\langle\dot{\sigma}_{0}, \ldots, \dot{\sigma}_{l-1}\right\rangle$ is the Weyl group of $U_{p}\left(\widehat{\mathfrak{s l}}_{l}\right)$ and so on. Similarly, if $M$ is a $U_{p}\left(\widehat{\mathfrak{s l}}_{l}\right)-$ module, denote by $\dot{\mathcal{P}}(M)$ the set of weights of $M$.

\subsection{The space $\Lambda^{s}$}

\subsubsection{The vector space $\Lambda^{s}$ and its standard basis}

Following [19], we now recall the definition of $\Lambda^{s}$, the space of semi-infinite $q$-wedge products of charge $s$ (this space is denoted by $\Lambda^{s+\frac{\infty}{2}}$ in [19]). First, let $r \geq 2$ be an 
integer, and $\Lambda_{q}^{r} V$ be the space of $q$-wedge products of finite length $r$ (this space is denoted by $\Lambda^{r}$ in [19]; we hope that this does not make any confusion with our $\Lambda^{s}$ ). As a vector space over $\mathbb{Q}(q), \Lambda_{q}^{r} V$ is spanned by the $q$-wedge products

$$
u_{\boldsymbol{k}}=u_{k_{1}} \wedge u_{k_{2}} \wedge \cdots \wedge u_{k_{r}}, \quad \boldsymbol{k}=\left(k_{1}, k_{2}, \ldots, k_{r}\right) \in \mathbb{Z}^{r},
$$

with relations given in [19, Prop. 3.16]. These relations are called straightening rules (we will not need them in this article). Now, define $\Lambda^{s}$ as the inductive limit

$$
\Lambda^{s}=\underset{\lim }{\longrightarrow} \Lambda_{q}^{r} V
$$

where maps $\Lambda_{q}^{r} V \rightarrow \Lambda_{q}^{r^{\prime}} V\left(r^{\prime}>r\right)$ are given by $v \mapsto v \wedge u_{s-r} \wedge u_{s-r-1} \wedge \cdots \wedge$ $u_{s-r^{\prime}+1}$. Less formally, $\Lambda^{s}$ is spanned by $q$-wedge products of infinite length

$$
u_{\boldsymbol{k}}=u_{k_{1}} \wedge u_{k_{2}} \wedge \cdots, \quad \boldsymbol{k}=\left(k_{1}, k_{2}, \ldots\right) \in P(s),
$$

where $P(s)$ is the set of all sequences of integers $\left(k_{1}, k_{2}, \ldots\right)$ such that $k_{i}=s-i+1$ for $i$ large enough. The straightening rules given in [19, Prop. 3.16 (i)] still hold for any pair of adjacent factors of a $q$-wedge product $u_{\boldsymbol{k}} \in \Lambda^{s}$. From now on, we shall assume without further comment that all $q$-wedge products lie in $\Lambda^{s}$ (in particular, they have infinitely many factors). Using the straightening rules, one can express a $q$-wedge product as a linear combination of so-called ordered $q$-wedge products, namely $q$-wedge products $u_{\boldsymbol{k}}$ with $\boldsymbol{k} \in P^{++}(s)$, where

$$
P^{++}(s):=\left\{\left(k_{1}, k_{2}, \ldots\right) \in P(s) \mid k_{1}>k_{2}>\cdots\right\} .
$$

In fact, the ordered $q$-wedge products $\left\{u_{\boldsymbol{k}} \mid \boldsymbol{k} \in P^{++}(s)\right\}$ form a basis of $\Lambda^{s}$, called the standard basis. In this article, it will be convenient to use different indexations of this basis which we give now.

* Indexation $u_{k}$. This is the indexation we have just described.

* Indexation $\lambda$. To the ordered $q$-wedge product $u_{k}$ corresponds a partition $\lambda=$ $\left(\lambda_{1}, \lambda_{2}, \ldots\right)$ defined by

$$
\lambda_{i}:=k_{i}-(s+1-i) \quad(i \geq 1) .
$$

If $u_{\boldsymbol{k}}$ and $\lambda$ are related this way, write

$$
|\lambda, s\rangle:=u_{k}
$$

* Indexation $\lambda_{n}$. Recall the definition of $\mathbb{Z}^{n}(s)$ from (1). Uglov constructed a bijection

$$
\tau_{n}^{\prime}: \Pi \rightarrow \Pi^{n} \times \mathbb{Z}^{n}(s), \quad \lambda \mapsto\left(\lambda_{n}, s_{n}\right)
$$

(see $[19, \S 4.1]$, where this map is denoted by $\tau_{n}^{s}$ ). With the notation above, $\lambda_{n}$ is the $n$-quotient of $\lambda$ and $s_{n}$ is a variation of the $n$-core of $\lambda$ (see e.g. [16, Ex.8, p.12]). Write

$$
\left|\lambda_{n}, s_{n}\right\rangle^{\bullet}:=|\lambda, s\rangle
$$


if $\left(\lambda_{n}, s_{n}\right)=\tau_{n}^{\prime}(\lambda)$. Note that this indexation coincides with the indexation $\lambda$ if $n=1$.

* Indexation $\lambda_{l}$. Uglov constructed a bijection

$$
\tau_{l}: \Pi \rightarrow \Pi^{l} \times \mathbb{Z}^{l}(s), \quad \lambda \mapsto\left(\lambda_{l}, s_{l}\right)
$$

(see again $[19, \S 4.1]$, where this map is denoted by $\tau_{l}^{s}$ ). The map $\tau_{l}$ is a variation of the map $\tau_{n}^{\prime}$ defined above. Write

$$
\left|\lambda_{l}, s_{l}\right\rangle:=|\lambda, s\rangle
$$

if $\left(\lambda_{l}, s_{l}\right)=\tau_{l}(\lambda)$. Note that this indexation coincides with the indexation $\lambda$ if $l=1$.

Example 2.1 Take $n=2, l=3$ and $s=-1$. Then we have

$$
\begin{aligned}
u_{3} \wedge u_{1} \wedge u_{0} \wedge u_{-2} \wedge u_{-4} \wedge u_{-6} \wedge u_{-7} \wedge \cdots & =|(4,3,3,2,1),-1\rangle \\
& =|((3,3), \varnothing),(-1,0)|^{\bullet} \\
& =\mid(1,1),(1,1),(1)),(0,0,-1)\rangle
\end{aligned}
$$

\subsubsection{Three actions on $\Lambda^{s}$}

Following [3, 6, 19], the vector space $\Lambda^{s}$ can be made into an integrable representation of level $l$ of the quantum algebra $U_{q}\left(\widehat{\mathfrak{s l}}_{n}\right)$. This representation can be described in a nice way if we use the indexation $\lambda_{l}$. In order to recall the explicit formulas, let us first introduce some notation. Fix $\lambda_{l}=\left(\lambda^{(1)}, \ldots, \lambda^{(l)}\right) \in \Pi^{l}$ and $s_{l}=\left(s_{1}, \ldots, s_{l}\right) \in \mathbb{Z}^{l}$. Identify the multi-partition $\lambda_{l}$ with its Young diagram $\left\{(i, j, b) \in \mathbb{N}^{*} \times \mathbb{N}^{*} \times \llbracket 1 ; l \rrbracket \mid 1 \leq j \leq \lambda_{i}^{(b)}\right\}$, whose elements are called nodes of $\lambda_{l}$. For each node $\gamma=(i, j, b)$ of $\lambda_{l}$, define its residue modulo $n$ by

$$
\operatorname{res}_{n}(\gamma)=\operatorname{res}_{n}\left(\gamma, s_{l}\right):=\left(s_{b}+j-i\right) \bmod n \in \mathbb{Z} / n \mathbb{Z} \cong \llbracket 0 ; n-1 \rrbracket .
$$

If $\operatorname{res}_{n}(\gamma)=c$, we say that $\gamma$ is a $c$-node. If $\mu_{l} \in \Pi^{l}$ is such that $\mu_{l} \supset \lambda_{l}$ and $\gamma:=$ $\boldsymbol{\mu}_{l} \backslash \lambda_{l}$ is a $c$-node of $\boldsymbol{\mu}_{l}$, we say that $\gamma$ is a removable c-node of $\boldsymbol{\mu}_{l}$ or that $\gamma$ is an addable c-node of $\lambda_{l}$. For $0 \leq c \leq n-1$, denote by

$$
M_{c}\left(\lambda_{l} ; s_{l} ; n\right) \quad\left(\text { respectively } A_{c}\left(\lambda_{l} ; s_{l} ; n\right), \quad \text { respectively } R_{c}\left(\lambda_{l} ; s_{l} ; n\right)\right)
$$

the number of $c$-nodes (respectively of addable $c$-nodes, respectively of removable $c$-nodes) of $\lambda_{l}$. Put

$$
N_{c}\left(\lambda_{l} ; s_{l} ; n\right):=A_{c}\left(\lambda_{l} ; s_{l} ; n\right)-R_{c}\left(\lambda_{l} ; s_{l} ; n\right) .
$$

For $\lambda_{l}, \mu_{l} \in \Pi^{l}, s_{l} \in \mathbb{Z}^{l}, c \in \llbracket 0 ; n-1 \rrbracket$ and $k \in \mathbb{N}^{*}$, write

$$
\lambda_{l} \stackrel{c: k}{\longrightarrow} \boldsymbol{\mu}_{l}
$$

if there exists a sequence of $l$-multi-partitions $\boldsymbol{v}_{l}^{(0)} \subset \boldsymbol{v}_{l}^{(1)} \subset \cdots \subset \boldsymbol{v}_{l}^{(k)}$ such that $\lambda_{l}=$ $\boldsymbol{v}_{l}^{(0)}, \boldsymbol{\mu}_{l}=\boldsymbol{v}_{l}^{(k)}$ and for all $1 \leq j \leq k, \boldsymbol{v}_{l}^{(j)} \backslash \boldsymbol{v}_{l}^{(j-1)}$ is an addable $c$-node of $\boldsymbol{v}_{l}^{(j-1)}$. 
Given a multi-charge $\left(s_{1}, \ldots, s_{l}\right)$ and two nodes $\gamma=(i, j, b)$ and $\gamma^{\prime}=\left(i^{\prime}, j^{\prime}, b^{\prime}\right)$, write

$$
\gamma<\gamma^{\prime}
$$

if either $s_{b}+j-i<s_{b^{\prime}}+j^{\prime}-i^{\prime}$ or $s_{b}+j-i=s_{b^{\prime}}+j^{\prime}-i^{\prime}$ and $b<b^{\prime}$. This defines a total ordering on the set of the addable and removable $c$-nodes of a given multi-partition. If $\lambda_{l} \stackrel{c: k}{\longrightarrow} \boldsymbol{\mu}_{l}$, put

$$
\begin{aligned}
N_{c}^{>}\left(\lambda_{l} ; \boldsymbol{\mu}_{l} ; \boldsymbol{s}_{l} ; n\right)= & \sum_{\gamma \in \mu_{l} \backslash \lambda_{l}}\left(\sharp\left\{\beta \in \mathbb{N}^{3} \mid \beta \text { is an addable } c \text {-node of } \boldsymbol{\mu}_{l} \text { and } \beta>\gamma\right\}\right. \\
& \left.-\sharp\left\{\beta \in \mathbb{N}^{3} \mid \beta \text { is a removable } c \text {-node of } \lambda_{l} \text { and } \beta>\gamma\right\}\right),(22)
\end{aligned}
$$

and define similarly $N_{c}^{<}\left(\boldsymbol{\lambda}_{l} ; \boldsymbol{\mu}_{l} ; \boldsymbol{s}_{l} ; n\right)$.

Example 2.2 Take $s_{l}=(5,0,2,1), \lambda_{l}=((5,3,3,1),(3,2),(4,3,1),(2,2,2,1))$, $n=3$ and $c=0$. Then we have

$$
M_{c}\left(\lambda_{l} ; s_{l} ; n\right)=11, \quad A_{c}\left(\lambda_{l} ; s_{l} ; n\right)=R_{c}\left(\lambda_{l} ; s_{l} ; n\right)=5 \quad \text { and } \quad N_{c}\left(\lambda_{l} ; s_{l} ; n\right)=0 .
$$

The addable $c$-nodes of $\lambda_{l}$ are $(5,1,4),(4,2,1),(1,4,2),(1,3,4)$ and $(1,5,3)$. The removable $c$-nodes of $\lambda_{l}$ are $(2,2,2),(3,1,3),(3,2,4),(2,3,3)$ and $(1,5,1)$. The list of all these nodes arranged with respect to the ordering described above is

$$
\begin{aligned}
(5,1,4) & <(2,2,2)<(3,1,3)<(3,2,4)<(4,2,1)<(1,4,2) \\
& <(2,3,3)<(1,3,4)<(1,5,3)<(1,5,1) .
\end{aligned}
$$

Take also $\boldsymbol{\mu}_{l}=((5,3,3,1),(3,2),(5,3,1),(2,2,2,1))$, so that $\mu_{l} \backslash \lambda_{l}=\{(1,5,3)\}$ is a single $c$-node. Then $N_{c}^{>}\left(\lambda_{l} ; \mu_{l} ; s_{l} ; n\right)=0-1=-1$ and $N_{c}^{<}\left(\lambda_{l} ; \mu_{l} ; s_{l} ; n\right)=$ $4-4=0$.

$$
\begin{aligned}
& \text { For } s_{l}=\left(s_{1}, \ldots, s_{l}\right) \in \mathbb{Z}^{l}, \text { define } \\
& \qquad \Delta\left(s_{l}, n\right):=\frac{1}{2} \sum_{b=1}^{l}\left(\frac{s_{b}^{2}}{n}-s_{b}\right)-\left(\frac{\left(s_{b} \bmod n\right)^{2}}{n}-\left(s_{b} \bmod n\right)\right) .
\end{aligned}
$$

Now we can state the following result.

Theorem 2.3 [3, 6, 19] The following formulas define on $\Lambda^{s}$ a structure of an integrable representation of level ll of the quantum algebra $U_{q}\left(\widehat{\mathfrak{s l}}_{n}\right)$.

$$
\begin{aligned}
& e_{i} \cdot\left|\boldsymbol{v}_{l}, \boldsymbol{s}_{l}\right\rangle= \sum_{\substack{\lambda_{l} \\
\stackrel{1: 1}{\longrightarrow} \boldsymbol{v}_{l}}} q^{-N_{i}^{<}\left(\lambda_{l} ; \boldsymbol{v}_{l} ; \boldsymbol{s}_{l} ; n\right)}\left|\lambda_{l}, \boldsymbol{s}_{l}\right\rangle, \\
& f_{i} \cdot\left|\boldsymbol{v}_{l}, \boldsymbol{s}_{l}\right\rangle=\sum_{\substack{i: 1 \\
\boldsymbol{v}_{l}}} q^{N_{i}^{>}\left(\boldsymbol{v}_{l} ; \boldsymbol{\mu}_{l} ; \boldsymbol{s}_{l} ; n\right)}\left|\boldsymbol{\mu}_{l}, \boldsymbol{s}_{l}\right\rangle,
\end{aligned}
$$




$$
\begin{aligned}
& t_{i} \cdot\left|\boldsymbol{v}_{l}, \boldsymbol{s}_{l}\right\rangle=q^{N_{i}\left(\boldsymbol{v}_{l} ; \boldsymbol{s}_{l} ; n\right)}\left|\boldsymbol{v}_{l}, \boldsymbol{s}_{l}\right\rangle, \\
& \text { ə.|}\left|\boldsymbol{v}_{l}, \boldsymbol{s}_{l}\right\rangle=-\left(\Delta\left(\boldsymbol{s}_{l}, n\right)+M_{0}\left(\boldsymbol{v}_{l} ; \boldsymbol{s}_{l} ; n\right)\right)\left|\boldsymbol{v}_{l}, \boldsymbol{s}_{l}\right\rangle .
\end{aligned}
$$

Note that these formulas involve no straightening of $q$-wedge products. They are therefore handy to use for computations.

In a completely similar way, $\Lambda^{s}$ can be made into an integrable representation of level $n$ of the quantum algebra $U_{p}\left(\widehat{\mathfrak{s l}}_{l}\right)$. This action can be described using the indexation $\lambda_{n}$. Namely, we have (with obvious notation) the following result.

Theorem 2.4 [3, 6, 19] The following formulas define on $\Lambda^{s}$ a structure of an integrable representation of level $n$ of the quantum algebra $U_{p}\left(\widehat{\mathfrak{s l}}_{l}\right)$.

$$
\begin{aligned}
& \dot{e}_{i} .\left|\boldsymbol{v}_{n}, \boldsymbol{s}_{n}\right\rangle^{\bullet}=\sum_{\substack{i: 1 \\
\lambda_{n} \stackrel{v_{n}}{\longrightarrow}}} p^{-N_{i}^{<}\left(\lambda_{n} ; \boldsymbol{v}_{n} ; \boldsymbol{s}_{n} ; l\right)}\left|\lambda_{n}, \boldsymbol{s}_{n}\right\rangle^{\bullet}, \\
& \dot{f}_{i} \cdot\left|\boldsymbol{v}_{n}, \boldsymbol{s}_{n}\right\rangle^{\bullet}=\sum_{\boldsymbol{v}_{n} \stackrel{i: 1}{\longrightarrow} \boldsymbol{\mu}_{n}} p^{N_{i}^{>}\left(\boldsymbol{v}_{n} ; \boldsymbol{\mu}_{n} ; \boldsymbol{s}_{n} ; l\right)}\left|\boldsymbol{\mu}_{n}, \boldsymbol{s}_{n}\right\rangle^{\bullet}, \\
& \dot{t}_{i} \cdot\left|\boldsymbol{v}_{n}, \boldsymbol{s}_{n}\right\rangle^{\bullet}=p^{N_{i}\left(\boldsymbol{v}_{n} ; \boldsymbol{s}_{n} ; l\right)}\left|\boldsymbol{v}_{n}, \boldsymbol{s}_{n}\right\rangle^{\bullet}, \\
& \dot{\partial} .\left|v_{n}, s_{n}\right\rangle^{\bullet}=-\left(\Delta\left(s_{n}, l\right)+M_{0}\left(v_{n} ; s_{n} ; l\right)\right)\left|v_{n}, s_{n}\right\rangle^{\bullet} .
\end{aligned}
$$

Theorems 2.3 and 2.4 show in particular that the vectors of the standard basis of $\Lambda^{s}$ are weight vectors for the actions of $U_{q}\left(\widehat{\mathfrak{s l}}_{n}\right)$ and $U_{p}\left(\widehat{\mathfrak{s l}}_{l}\right)$, and the weights are given by:

Corollary 2.5 [19], (27-30) With obvious notation, we have

$$
\begin{aligned}
\operatorname{wt}\left(\left|\lambda_{l}, s_{l}\right\rangle\right)= & -\Delta\left(s_{l}, n\right) \delta+\Lambda_{s_{1}}+\cdots+\Lambda_{s_{l}}-\sum_{i=0}^{n-1} M_{i}\left(\lambda_{l} ; s_{l} ; n\right) \alpha_{i}, \\
\dot{\operatorname{wt}}\left(\left|\lambda_{l}, s_{l}\right\rangle\right)= & -\left(\Delta\left(s_{l}, n\right)+M_{0}\left(\lambda_{l} ; s_{l} ; n\right)\right) \dot{\delta}+\left(n-s_{1}+s_{l}\right) \dot{\Lambda}_{0} \\
& +\sum_{i=1}^{l-1}\left(s_{i}-s_{i+1}\right) \dot{\Lambda}_{i}, \\
\dot{\operatorname{wt}}\left(\left|\lambda_{n}, s_{n}\right\rangle^{\bullet}\right)= & -\Delta\left(s_{n}, l\right) \dot{\delta}+\dot{\Lambda}_{s_{1}}+\cdots+\dot{\Lambda}_{s_{n}}-\sum_{i=0}^{l-1} M_{i}\left(\lambda_{n} ; s_{n} ; l\right) \dot{\alpha}_{i},
\end{aligned}
$$




$$
\begin{aligned}
\operatorname{wt}\left(\left|\lambda_{n}, s_{n}\right\rangle^{\bullet}\right)= & -\left(\Delta\left(s_{n}, l\right)+M_{0}\left(\lambda_{n} ; s_{n} ; l\right)\right) \delta+\left(l-s_{1}+s_{n}\right) \Lambda_{0} \\
& +\sum_{i=1}^{n-1}\left(s_{i}-s_{i+1}\right) \Lambda_{i} .
\end{aligned}
$$

Definition 2.6 For $m \in \mathbb{Z}^{*}$, define an endomorphism $B_{m}$ of $\Lambda^{s}$ by

$$
\begin{aligned}
& B_{m}\left(u_{k_{1}} \wedge u_{k_{2}} \wedge \cdots\right):=\sum_{j=1}^{+\infty} u_{k_{1}} \wedge \cdots \wedge u_{k_{j-1}} \wedge u_{k_{j}-n l m} \wedge u_{k_{j+1}} \wedge \cdots \\
& \quad\left(\left(k_{1}, k_{2}, \ldots\right) \in P^{++}(s)\right) .
\end{aligned}
$$

Using a variation of [19, Lemma 3.18] for $q$-wedge products with infinitely many factors, one sees that the sum above involves only finitely many nonzero terms, hence $B_{m}$ is well-defined. This definition comes from a passage to the limit $r \rightarrow \infty$ in the action of the center of the Hecke algebra of $\widehat{\mathfrak{S}}_{r}$ on $q$-wedge products of $r$ factors. However, the operators $B_{m}$ do not commute, but by [19, Prop. 4.4], they span a Heisenberg algebra

$$
\mathcal{H}:=\left\langle B_{m} \mid m \in \mathbb{Z}^{*}\right\rangle .
$$

We now recall some results concerning the actions of $U_{q}\left(\widehat{\mathfrak{s l}}_{n}\right), U_{p}\left(\widehat{\mathfrak{s l}}_{l}\right)$ and $\mathcal{H}$ on $\Lambda^{s}$.

Proposition 2.7 [19], Prop. 4.6 Recall that $p=-q^{-1}$. Then the actions of $U_{q}^{\prime}\left(\widehat{\mathfrak{s l}}_{n}\right)$, $U_{p}^{\prime}\left(\widehat{\mathfrak{s l}}_{l}\right)$ and $\mathcal{H}$ on $\Lambda^{s}$ pairwise commute.

For $L, N \in \mathbb{N}^{*}$, introduce the finite set

$$
A_{L, N}(s):=\left\{\left(r_{1}, \ldots, r_{L}\right) \in \mathbb{Z}^{L}(s) \mid r_{1} \geq \cdots \geq r_{L}, r_{1}-r_{L} \leq N\right\} .
$$

Using [19, §4.1], it is not hard to see that if $\boldsymbol{r}_{l} \in \mathbb{Z}^{l}(s)$ and $\boldsymbol{r}_{n} \in \mathbb{Z}^{n}(s)$ are such that $\left|\emptyset_{l}, \boldsymbol{r}_{l}\right\rangle=\left|\emptyset_{n}, \boldsymbol{r}_{n}\right\rangle^{\bullet}$, then $\boldsymbol{r}_{l} \in A_{l, n}(s)$ and $\boldsymbol{r}_{n} \in A_{n, l}(s)$. Conversely, if $\boldsymbol{r}_{l} \in A_{l, n}(s)$, then there exists a unique $\boldsymbol{r}_{n} \in A_{n, l}(s)$ such that $\left|\emptyset_{n}, \boldsymbol{r}_{n}\right\rangle^{\bullet}=\left|\emptyset_{l}, \boldsymbol{r}_{l}\right\rangle$, and if $\boldsymbol{r}_{n} \in$ $A_{n, l}(s)$, then there exists a unique $\boldsymbol{r}_{l} \in A_{l, n}(s)$ such that $\left|\emptyset_{l}, \boldsymbol{r}_{l}\right\rangle=\left|\emptyset_{n}, \boldsymbol{r}_{n}\right\rangle^{\bullet}$. Therefore,

$$
\left\{\left|\emptyset_{l}, \boldsymbol{r}_{l}\right\rangle \mid \boldsymbol{r}_{l} \in A_{l, n}(s)\right\}=\left\{\left|\emptyset_{n}, \boldsymbol{r}_{n}\right\rangle \bullet \mid \boldsymbol{r}_{n} \in A_{n, l}(s)\right\}
$$

is a set of highest weight vectors simultaneously for the actions of $U_{q}^{\prime}\left(\widehat{\mathfrak{s l}}_{n}\right)$ and $U_{p}^{\prime}\left(\widehat{\mathfrak{s l}}_{l}\right)$. It is easy to see that these vectors are also singular for the action of $\mathcal{H}$, that is, they are annihilated by the $B_{m}, m>0$. It turns out that these vectors are the only singular vectors simultaneously for the actions of $U_{q}^{\prime}\left(\widehat{\mathfrak{s l}}_{n}\right), U_{p}^{\prime}\left(\widehat{\mathfrak{s l}}_{l}\right)$ and $\mathcal{H}$, and we have the following theorem. 
Theorem 2.8 [19], Thm. 4.8 We have

$$
\begin{aligned}
\Lambda^{s} & =\bigoplus_{r_{l} \in A_{l, n}(s)} U_{q}^{\prime}\left(\widehat{\mathfrak{s l}}_{n}\right) \otimes \mathcal{H} \otimes U_{p}^{\prime}\left(\widehat{\mathfrak{s l}}_{l}\right) \cdot\left|\emptyset_{l}, \boldsymbol{r}_{l}\right\rangle \\
& =\bigoplus_{\boldsymbol{r}_{n} \in A_{n, l}(s)} U_{q}^{\prime}\left(\widehat{\mathfrak{s l}}_{n}\right) \otimes \mathcal{H} \otimes U_{p}^{\prime}\left(\widehat{\mathfrak{s l}}_{l}\right) \cdot\left|\emptyset_{n}, \boldsymbol{r}_{n}\right\rangle^{\bullet} .
\end{aligned}
$$

\subsubsection{The involution - of $\Lambda^{s}$}

Following [19], the space $\Lambda^{s}$ can be endowed with an involution - . Instead of recalling the definition of this involution, we give its main properties (by [23, Thm. 3.11], they turn out to characterize it completely).

Proposition 2.9 [19] There exists an involution - of $\Lambda^{s}$ such that:

(i) - is a $\mathbb{Q}$-linear map of $\Lambda^{s}$ such that for all $u \in \Lambda^{s}, k \in \mathbb{Z}$, we have $\overline{q^{k} u}=q^{-k} \bar{u}$.

(ii) (Unitriangularity property). For all $\lambda \in \Pi$, we have

$$
\overline{|\lambda, s\rangle} \in|\lambda, s\rangle+\bigoplus_{\mu \triangleleft \lambda} \mathbb{Z}\left[q, q^{-1}\right]|\mu, s\rangle,
$$

where $\triangleleft$ stands for the dominance ordering on partitions.

(iii) For all $\lambda \in \Pi$, we have $\mathrm{wt}(\overline{|\lambda, s\rangle})=\mathrm{wt}(|\lambda, s\rangle)$ and $\dot{\mathrm{w}} \mathrm{t}(\overline{|\lambda, s\rangle})=\dot{\mathrm{w}} \mathrm{t}(|\lambda, s\rangle)$.

(iv) For all $0 \leq i \leq n-1,0 \leq j \leq l-1, m<0, v \in \Lambda^{s}$, we have

$$
\overline{f_{i} \cdot v}=f_{i} \cdot \bar{v}, \quad \overline{\dot{f}_{j} \cdot v}=\dot{f}_{j} \cdot \bar{v} \quad \text { and } \quad \overline{B_{m} \cdot v}=B_{m} \cdot \bar{v} .
$$

Proof Let - be the involution of $\Lambda^{s}$ defined in [19, Prop. $3.23 \&$ Eqn. (39)]. By construction, (i) holds. The other statements come from [19, Prop. $4.11 \& 4.12]$ and Corollary 2.5.

\section{$2.3 q$-deformed higher-level Fock spaces}

\subsubsection{Definition}

By Theorem 2.3, the space

$$
\mathbf{F}_{q}\left[s_{l}\right]:=\bigoplus_{\lambda_{l} \in \Pi^{l}} \mathbb{Q}(q)\left|\lambda_{l}, s_{l}\right\rangle \subset \Lambda^{s} \quad\left(s_{l} \in \mathbb{Z}^{l}(s)\right)
$$

is a $U_{q}\left(\widehat{\mathfrak{s l}}_{n}\right)$-submodule of $\Lambda^{s}$. The reader should be aware that $\mathbf{F}_{q}\left[\boldsymbol{s}_{l}\right]$ is not a $U_{p}\left(\widehat{\mathfrak{s l}}_{l}\right)$-submodule of $\Lambda^{s}$. In a similar way, by Theorem 2.4 , the space

$$
\mathbf{F}_{p}\left[\boldsymbol{s}_{n}\right]^{\bullet}:=\bigoplus_{\lambda_{n} \in \Pi^{n}} \mathbb{Q}(q)\left|\lambda_{n}, \boldsymbol{s}_{n}\right\rangle^{\bullet} \subset \Lambda^{s} \quad\left(\boldsymbol{s}_{n} \in \mathbb{Z}^{n}(s)\right)
$$

is a $U_{p}\left(\widehat{\mathfrak{s l}}_{l}\right)$-submodule of $\Lambda^{s}$. 
Definition 2.10 [19] The representations $\mathbf{F}_{q}\left[\boldsymbol{s}_{l}\right]$ and $\mathbf{F}_{p}\left[\boldsymbol{s}_{n}\right]^{\bullet}\left(\boldsymbol{s}_{l} \in \mathbb{Z}^{l}(s), \boldsymbol{s}_{n} \in \mathbb{Z}^{n}(s)\right)$ are called (q-deformed) Fock spaces. When $l>1$ and $n>1$, we speak of higher-level Fock spaces.

Since the maps $\tau_{l}$ and $\tau_{n}^{\prime}$ are bijections, we have the following decompositions:

$$
\Lambda^{s}=\bigoplus_{s_{l} \in \mathbb{Z}^{l}(s)} \mathbf{F}_{q}\left[s_{l}\right]=\bigoplus_{s_{n} \in \mathbb{Z}^{n}(s)} \mathbf{F}_{p}\left[\boldsymbol{s}_{n}\right]^{\bullet} .
$$

Neither of these decompositions is compatible with the decompositions of $\Lambda^{s}$ given in Theorem 2.8.

\subsubsection{Fock spaces as weight subspaces of $\Lambda^{s}$. Actions of the Weyl groups.}

Let $N, L \in \mathbb{N}^{*}$. Recall the definition of $\mathbb{Q}^{L}(s)$ and $\mathbb{Q}^{L}(N)$ from (1) and define a map

$$
\theta_{L, N}: \mathbb{Q}^{L}(s) \rightarrow \mathbb{Q}^{L}(N), \quad\left(s_{1}, \ldots, s_{L}\right) \mapsto\left(N-s_{1}+s_{L}, s_{1}-s_{2}, \ldots, s_{L-1}-s_{L}\right) .
$$

(Note that this map also depends on the charge $s \in \mathbb{Z}$ that we have fixed. However, since $s$ will not vary in this paper, we do not keep it in our notation.) It is easy to see that $\theta_{L, N}$ is bijective. Moreover, for $\left(a_{1}, \ldots, a_{L}\right) \in \mathbb{Q}^{L}(N)$, the $l$-tuple $\left(s_{1}, \ldots, s_{L}\right):=\theta_{L, N}^{-1}\left(a_{1}, \ldots, a_{L}\right)$ is given by

$$
s_{i}=\frac{1}{L}\left(s-\sum_{j=1}^{L-1} j a_{j+1}\right)+\sum_{j=i+1}^{L} a_{j} \quad(1 \leq i \leq L) .
$$

The next result shows that the Fock spaces are sums of certain weight subspaces of $\Lambda^{s}$. The proof follows easily from Corollary 2.5 .

\section{Proposition 2.11 [19]}

(i) Let $\boldsymbol{s}_{n} \in \mathbb{Z}^{n}(s)$. Let $\left(a_{1}, \ldots, a_{n}\right):=\theta_{n, l}\left(\boldsymbol{s}_{n}\right)$ and $w:=\sum_{i=1}^{n} a_{i} \Lambda_{i-1}$. Then

$$
\mathbf{F}_{p}\left[\boldsymbol{s}_{n}\right]^{\bullet}=\bigoplus_{d \in \mathbb{Z}} \Lambda^{s}\langle w+d \delta\rangle
$$

(ii) Let $s_{l} \in \mathbb{Z}^{l}(s)$. Let $\left(a_{1}, \ldots, a_{l}\right):=\theta_{l, n}\left(s_{l}\right)$ and $\dot{w}:=\sum_{i=1}^{l} a_{i} \dot{\Lambda}_{i-1}$. Then

$$
\mathbf{F}_{q}\left[s_{l}\right]=\bigoplus_{d \in \mathbb{Z}} \Lambda^{s}\langle\dot{w}+d \dot{\delta}\rangle
$$

Note that the operator $B_{m}\left(m \in \mathbb{Z}^{*}\right)$ maps the weight subspace $\Lambda^{s}\langle w\rangle$ (respectively $\Lambda^{s}\langle\dot{w}\rangle$ ) into $\Lambda^{s}\langle w+m \delta\rangle$ (respectively $\Lambda^{s}\langle\dot{w}+m \dot{\delta}\rangle$ ). Therefore, by Proposition 2.11, the Fock spaces $\mathbf{F}_{q}\left[\boldsymbol{s}_{l}\right]$ and $\mathbf{F}_{p}\left[\boldsymbol{s}_{n}\right]^{\bullet}\left(\boldsymbol{s}_{l} \in \mathbb{Z}^{l}(s), \boldsymbol{s}_{n} \in \mathbb{Z}^{n}(s)\right)$ are stable under the action of $\mathcal{H}$. 
We now compare some weight subspaces of the Fock spaces. The proof follows again from Corollary 2.5 .

\section{Proposition 2.12}

(i) Let $\boldsymbol{s}_{l}=\left(s_{1}, \ldots, s_{l}\right) \in \mathbb{Z}^{l}(s)$ and $w$ be a weight of $\mathbf{F}_{q}\left[\boldsymbol{s}_{l}\right]$. Then there exists a unique pair $\left(\boldsymbol{s}_{n}, \dot{w}\right)$ such that $\mathbf{F}_{q}\left[\boldsymbol{s}_{l}\right]\langle w\rangle=\mathbf{F}_{p}\left[\boldsymbol{s}_{n}\right]^{\bullet}\langle\dot{w}\rangle$, where $\boldsymbol{s}_{n}$ is in $\mathbb{Z}^{n}(s)$ and $\dot{w}$ is a weight of $\mathbf{F}_{p}\left[s_{n}\right]^{\bullet}$. More precisely, write $w=d \delta+\sum_{i=1}^{n} a_{i} \Lambda_{i-1}$ with $a_{1}, \ldots, a_{n}, d \in \mathbb{Z}$, and put $s_{0}:=n+s_{l}$. Then we have $s_{n}=\theta_{n, l}^{-1}\left(a_{1}, \ldots, a_{n}\right)$ and $\dot{w}=d \dot{\delta}+\sum_{i=0}^{l-1}\left(s_{i}-s_{i+1}\right) \dot{\Lambda}_{i}$.

(ii) Let $\boldsymbol{s}_{n}=\left(s_{1}, \ldots, s_{n}\right) \in \mathbb{Z}^{n}(s)$ and $\dot{w}$ be a weight of $\mathbf{F}_{p}\left[\boldsymbol{s}_{n}\right]^{\bullet}$. Then there exists a unique pair $\left(\boldsymbol{s}_{l}, w\right)$ such that $\mathbf{F}_{p}\left[\boldsymbol{s}_{n}\right]^{\bullet}\langle\dot{w}\rangle=\mathbf{F}_{q}\left[\boldsymbol{s}_{l}\right]\langle w\rangle$, where $\boldsymbol{s}_{l}$ is in $\mathbb{Z}^{l}(s)$ and $w$ is a weight of $\mathbf{F}_{q}\left[s_{l}\right]$. More precisely, write $\dot{w}=d \dot{\delta}+\sum_{i=1}^{l} a_{i} \dot{\Lambda}_{i-1}$ with $a_{1}, \ldots, a_{l}, d \in \mathbb{Z}$, and put $s_{0}:=l+s_{n}$. Then we have $s_{l}=\theta_{l, n}^{-1}\left(a_{1}, \ldots, a_{l}\right)$ and $w=d \delta+\sum_{i=0}^{n-1}\left(s_{i}-s_{i+1}\right) \Lambda_{i}$.

Example 2.13 Take $n=3, l=2, s_{l}=(1,0)$ and $w=-2 \Lambda_{0}+\Lambda_{1}+3 \Lambda_{2}-2 \delta$. Then by (32), we have $\operatorname{wt}\left(\left|((1,1),(1)), s_{l}\right\rangle\right)=w$, so $w$ is a weight of $\mathbf{F}_{q}\left[s_{l}\right]$. By Proposition 2.12 (i), we have $\mathbf{F}_{q}\left[\boldsymbol{s}_{l}\right]\langle w\rangle=\mathbf{F}_{p}\left[\boldsymbol{s}_{n}\right]^{\bullet}\langle\dot{w}\rangle$ with $\boldsymbol{s}_{n}=(2,1,-2)$ and $\dot{w}=2 \dot{\Lambda}_{0}+\dot{\Lambda}_{1}-2 \dot{\delta}$. Moreover, using (32) and (34), we see that for all $\left|\lambda_{l}, s_{l}\right\rangle=$ $\left|\lambda_{n}, s_{n}\right\rangle^{\bullet} \in \mathbf{F}_{q}\left[s_{l}\right]\langle w\rangle=\mathbf{F}_{p}\left[s_{n}\right]^{\bullet}\langle\dot{w}\rangle$, we have $M_{0}\left(\lambda_{l} ; s_{l} ; n\right)=2, M_{1}\left(\lambda_{l} ; s_{l} ; n\right)=1$, $M_{2}\left(\lambda_{l} ; s_{l} ; n\right)=0$ and $M_{0}\left(\lambda_{n} ; s_{n} ; l\right)=M_{1}\left(\lambda_{n} ; s_{n} ; l\right)=0$ (this shows a posteriori that $\left.\operatorname{dim}\left(\mathbf{F}_{q}\left[s_{l}\right]\langle w\rangle\right)=1\right)$.

We now deal with the actions of the Weyl groups of $U_{q}\left(\widehat{\mathfrak{s l}}_{n}\right)$ and $U_{p}\left(\widehat{\mathfrak{s l}}_{l}\right)$ on the set of the weight subspaces of $\Lambda^{s}$. Recall that $W_{n}=\left\langle\sigma_{0}, \ldots, \sigma_{n-1}\right\rangle$ is the Weyl group of $U_{q}\left(\widehat{\mathfrak{s l}}_{n}\right)$. Since the $\alpha_{i}$ 's are the simple roots and the $\Lambda_{j}$ 's are the fundamental weights for the Kac-Moody algebra $\widehat{\mathfrak{s l}}_{n}$, we have $\left(\Lambda_{j}, \alpha_{i}\right)=\delta_{i, j}$ for all $0 \leq i, j \leq n-1$. Hence by (5), $W_{n}$ acts on the weight lattice $\bigoplus_{i=0}^{n-1} \mathbb{Z} \Lambda_{i} \oplus \mathbb{Z} \delta$ by

$$
\begin{aligned}
& \sigma_{i} . \delta=\delta \quad \text { and } \\
& \sigma_{i} \cdot \Lambda_{j}=\left\{\begin{array}{ll}
\Lambda_{j} & \text { if } j \neq i, \\
\Lambda_{i-1}+\Lambda_{i+1}-\Lambda_{i}-\delta_{i, 0} \delta & \text { if } j=i
\end{array} \quad(0 \leq i, j \leq n-1) .\right.
\end{aligned}
$$

Moreover, it is easy to see that $W_{n}$ acts faithfully on $\mathbb{Z}^{n}(s)$ by

$$
\left\{\begin{array}{l}
\sigma_{0} \cdot\left(s_{1}, \ldots, s_{n}\right)=\left(s_{n}+l, s_{2}, \ldots, s_{n-1}, s_{1}-l\right), \\
\sigma_{i} .\left(s_{1}, \ldots, s_{n}\right)=\left(s_{1}, \ldots, s_{i+1}, s_{i}, \ldots, s_{n}\right)
\end{array}(1 \leq i \leq n-1),\right.
$$

and the set $A_{n, l}(s)$ defined by (38) is a fundamental domain for this action. In a similar way, one can define two actions of the Weyl group $\dot{W}_{l}$ of $U_{p}\left(\widehat{\mathfrak{s l}}_{l}\right)$, one on the weight lattice $\bigoplus_{i=0}^{l-1} \mathbb{Z} \dot{\Lambda}_{i} \oplus \mathbb{Z} \dot{\delta}$ and one on $\mathbb{Z}^{l}(s)$. The following lemma will be useful later.

Lemma 2.14 Let $\boldsymbol{s}_{n} \in \mathbb{Z}^{n}(s)$ and $\dot{w}$ be a weight of $\mathbf{F}_{p}\left[s_{n}\right]^{\bullet}$. Let $\left(s_{l}, w\right) \in \mathbb{Z}^{l}(s) \times$ $\mathcal{P}\left(\Lambda^{s}\right)$ be the unique pair such that $\mathbf{F}_{p}\left[\boldsymbol{s}_{n}\right]^{\bullet}\langle\dot{w}\rangle=\mathbf{F}_{q}\left[s_{l}\right]\langle w\rangle$ (see Proposition 2.12 
(i)). Let $\dot{\sigma} \in \dot{W}_{l}$. In the same way, let $\left(\boldsymbol{t}_{l}, w^{\prime}\right) \in \mathbb{Z}^{l}(s) \times \mathcal{P}\left(\Lambda^{s}\right)$ be the unique pair such that $\mathbf{F}_{p}\left[\boldsymbol{s}_{n}\right]^{\bullet}\langle\dot{\sigma} . \dot{w}\rangle=\mathbf{F}_{q}\left[\boldsymbol{t}_{l}\right]\left\langle w^{\prime}\right\rangle$. Then we have

$$
\boldsymbol{t}_{l}=\dot{\sigma} \cdot s_{l} \quad \text { and } \quad w^{\prime}=w+\operatorname{wt}\left(\left|\emptyset_{l}, \boldsymbol{t}_{l}\right\rangle\right)-\operatorname{wt}\left(\left|\emptyset_{l}, s_{l}\right\rangle\right) .
$$

Proof The proof follows immediately from the formulas given in Proposition 2.12.

\subsubsection{The lower crystal basis $\left(\mathcal{L}\left[\boldsymbol{s}_{l}\right], \mathcal{B}\left[\boldsymbol{s}_{l}\right]\right)$ of $\mathbf{F}_{q}\left[\boldsymbol{s}_{l}\right]$ at $q=0$}

Let $s_{l} \in \mathbb{Z}^{l}(s)$. Following [8], let $\mathbb{A} \subset \mathbb{Q}(q)$ be the ring of rational functions which are regular at $q=0, \mathcal{L}\left[s_{l}\right]:=\bigoplus_{\lambda_{l} \in \Pi^{l}} \mathbb{A}\left|\lambda_{l}, s_{l}\right\rangle$ and for $0 \leq i \leq n-1$, let $\tilde{e}_{i}^{\text {low }}, \tilde{f}_{i}^{\text {low }}$, $\tilde{e}_{i}$ up and $\tilde{f}_{i}{ }^{\text {up }}$ denote Kashiwara's operators acting on $\mathcal{L}\left[s_{l}\right]$. The following lemma shows that sometimes, certain powers of the operators ${\tilde{e_{i}}}^{\text {low }}$ and ${\tilde{e_{i}}}^{\text {up }}$ coincide (one has an analogous result for $\tilde{f}_{i}^{\text {low }}$ and $\tilde{f}_{i}^{\text {up }}$ ).

Lemma 2.15 Let $s_{l} \in \mathbb{Z}^{l}(s), w \in \mathcal{P}\left(\mathbf{F}_{q}\left[s_{l}\right]\right), u \in\left(\operatorname{Ker} e_{i}\right) \cap \mathbf{F}_{q}\left[s_{l}\right]\langle w\rangle$ and $k:=$ $\left(w, \alpha_{i}\right)$. Then we have

$$
\left(\tilde{e}_{i}^{\text {up }}\right)^{k} \cdot\left(f_{i}^{(k)} \cdot u\right)=\left(\tilde{e}_{i}^{\text {low }}\right)^{k} \cdot\left(f_{i}^{(k)} \cdot u\right)=u .
$$

Proof The second equality follows easily by induction on $k$ from the definition of ${\tilde{e_{i}}}^{\text {low }}$. Let us now show that $\left({\tilde{e_{i}}}^{\text {up }}\right)^{k} \cdot\left(f_{i}^{(k)} \cdot u\right)=u$. Note that for $0 \leq j \leq k$, we have

$$
\left(\operatorname{wt}\left(f_{i}^{(k-j)} \cdot u\right), \alpha_{i}\right)=\left(\operatorname{wt}(u), \alpha_{i}\right)-(k-j)\left(\alpha_{i}, \alpha_{i}\right)=2 j-k
$$

By induction on $0 \leq k^{\prime} \leq k$, we get therefore, by definition of ${\tilde{e_{i}}}^{\text {up }}$,

$$
\left(\tilde{e}_{i}^{\text {up }}\right)^{k^{\prime}} \cdot\left(f_{i}^{(k)} \cdot u\right)=\left(\prod_{j=0}^{k^{\prime}-1} \frac{[(2 j-k)+(k-j)+1]}{[k-j]}\right) f_{i}^{\left(k-k^{\prime}\right)} \cdot u \quad\left(0 \leq k^{\prime} \leq k\right) .
$$

As a consequence, we have $\left(\tilde{e}_{i}^{\text {up }}\right)^{k} \cdot\left(f_{i}^{(k)} \cdot u\right)=\left(\prod_{j=0}^{k-1} \frac{[j+1]}{[k-j]}\right) u=u$.

Put

$$
\mathcal{B}\left[s_{l}\right]:=\left\{\left|\lambda_{l}, s_{l}\right\rangle \bmod q \mathcal{L}\left[s_{l}\right] \mid \lambda_{l} \in \Pi^{l}\right\}
$$

From now on, we shall write more briefly $\lambda_{l}$ for the element in $\mathcal{B}\left[s_{l}\right]$ indexed by the corresponding multi-partition. By $[3,6,19]$, the pair $\left(\mathcal{L}\left[s_{l}\right], \mathcal{B}\left[s_{l}\right]\right)$ is a lower crystal basis of $\mathbf{F}_{q}\left[s_{l}\right]$ at $q=0$ in the sense of [8], and the crystal graph $\mathcal{B}\left[\boldsymbol{s}_{l}\right]$ contains the arrow $\lambda_{l} \stackrel{i}{\longrightarrow} \mu_{l}$ if and only if the multi-partition $\mu_{l}$ is obtained from $\lambda_{l}$ by adding a good $i$-node in the sense of [19, Thm. 2.4]. We shall still denote by $\tilde{e}_{i}^{\text {low }}$ and $\tilde{f}_{i}^{\text {low }}$ Kashiwara's operators acting on $\mathcal{B}\left[\boldsymbol{s}_{l}\right] \cup\{0\}$. 


\subsubsection{Uglov's canonical bases of the Fock spaces}

By Propositions 2.9 (iii) and 2.11, the Fock spaces $\mathbf{F}_{q}\left[\boldsymbol{s}_{l}\right]$ and $\mathbf{F}_{p}\left[\boldsymbol{s}_{n}\right]^{\bullet}\left(\boldsymbol{s}_{l} \in \mathbb{Z}^{l}(s)\right.$, $s_{n} \in \mathbb{Z}^{n}(s)$ ) are stable under the involution - . The involution induced on these spaces will still be denoted by ${ }^{-}$. Let $\boldsymbol{s}_{l} \in \mathbb{Z}^{l}(s)$. For $\boldsymbol{\mu}_{l} \in \Pi^{l}$, write

$$
\overline{\left|\mu_{l}, s_{l}\right\rangle}=\sum_{\lambda_{l} \in \Pi^{l}} a_{\lambda_{l}, \mu_{l} ; s_{l}}(q)\left|\lambda_{l}, s_{l}\right\rangle
$$

with $a_{\lambda_{l}, \mu_{l} ; s_{l}}(q) \in \mathbb{Z}\left[q, q^{-1}\right]$, and let

$$
A_{\boldsymbol{s}_{l}}(q):=\left(a_{\lambda_{l}, \boldsymbol{\mu}_{l} ; s_{l}}(q)\right)_{\lambda_{l}, \boldsymbol{\mu}_{l} \in \Pi^{l}}
$$

denote the matrix of the involution ${ }^{-}$of $\mathbf{F}_{q}\left[\boldsymbol{s}_{l}\right]$. Since the weight subspaces of $\mathbf{F}_{q}\left[\boldsymbol{s}_{l}\right]$ are stable under the involution - , (32) implies that $a_{\lambda_{l}, \boldsymbol{\mu}_{l} ; \boldsymbol{s}_{l}}(q)$ is zero unless $\left|\lambda_{l}\right|=$ $\left|\boldsymbol{\mu}_{l}\right|$, where $\left|\lambda_{l}\right|$ (respectively $\left|\boldsymbol{\mu}_{l}\right|$ ) denotes the number of boxes contained in the Young diagram of $\left|\lambda_{l}\right|$ (respectively $\left|\boldsymbol{\mu}_{l}\right|$ ). By Proposition 2.9 (ii), the matrix $A_{\boldsymbol{s}_{l}}(q)$ is unitriangular. One can therefore define, by a classical argument, canonical bases of the Fock space $\mathbf{F}_{q}\left[\boldsymbol{s}_{l}\right]$ as follows.

Theorem 2.16 [19] Let $\boldsymbol{s}_{l} \in \mathbb{Z}^{l}(s)$. Then there exists a unique base

$$
\left\{G^{+}\left(\lambda_{l}, s_{l}\right) \mid \lambda_{l} \in \Pi^{l}\right\} \quad\left(\text { respectively }\left\{G^{-}\left(\lambda_{l}, s_{l}\right) \mid \lambda_{l} \in \Pi^{l}\right\}\right)
$$

of $\mathbf{F}_{q}\left[\boldsymbol{s}_{l}\right]$ such that:

(i) $\overline{G^{+}\left(\lambda_{l}, s_{l}\right)}=G^{+}\left(\lambda_{l}, s_{l}\right) \quad$ (respectively $\left.\overline{G^{-}\left(\lambda_{l}, s_{l}\right)}=G^{-}\left(\lambda_{l}, s_{l}\right)\right)$,

(ii) $G^{+}\left(\lambda_{l}, s_{l}\right) \equiv\left|\lambda_{l}, s_{l}\right\rangle \bmod q \mathcal{L}^{+}\left[s_{l}\right]$ (respectively $G^{-}\left(\lambda_{l}, s_{l}\right) \equiv\left|\lambda_{l}, s_{l}\right\rangle$ $\left.\bmod q^{-1} \mathcal{L}^{-}\left[s_{l}\right]\right)$,

where $\mathcal{L}^{\epsilon}\left[s_{l}\right]:=\bigoplus_{\lambda_{l} \in \Pi^{l}} \mathbb{Z}\left[q^{\epsilon}\right]\left|\lambda_{l}, s_{l}\right\rangle(\epsilon= \pm 1)$

Definition 2.17 The bases $\left\{G^{+}\left(\lambda_{l}, s_{l}\right) \mid \lambda_{l} \in \Pi^{l}\right\}$ and $\left\{G^{-}\left(\lambda_{l}, s_{l}\right) \mid \lambda_{l} \in \Pi^{l}\right\}$ are called Uglov's canonical bases of $\mathbf{F}_{q}\left[s_{l}\right]$. Define entries $\Delta_{\lambda_{l}, \mu_{l} ; s_{l}}^{+}(q), \Delta_{\lambda_{l}, \mu_{l} ; s_{l}}^{-}(q) \in$ $\mathbb{Z}\left[q, q^{-1}\right]\left(\lambda_{l}, \mu_{l} \in \Pi^{l}\right)$ by

$$
G^{+}\left(\mu_{l}, s_{l}\right)=\sum_{\lambda_{l} \in \Pi^{l}} \Delta_{\lambda_{l}, \mu_{l} ; s_{l}}^{+}(q)\left|\lambda_{l}, s_{l}\right\rangle, \quad G^{-}\left(\mu_{l}, s_{l}\right)=\sum_{\lambda_{l} \in \Pi^{l}} \Delta_{\lambda_{l}, \mu_{l} ; s_{l}}^{-}(q)\left|\lambda_{l}, s_{l}\right\rangle,
$$

and denote by

$$
\Delta_{\boldsymbol{s}_{l}}^{\epsilon}(q):=\left(\Delta_{\lambda_{l}, \boldsymbol{\mu}_{l} ; \boldsymbol{s}_{l}}^{\epsilon}(q)\right)_{\lambda_{l}, \boldsymbol{\mu}_{l} \in \Pi^{l}} \quad(\epsilon= \pm 1)
$$

the transition matrices between the standard and the canonical bases of $\mathbf{F}_{q}\left[\boldsymbol{s}_{l}\right]$. 
By [19], the entries of $\Delta_{\boldsymbol{s}_{l}}^{+}(q)$ (respectively $\Delta_{\boldsymbol{s}_{l}}^{-}(q)$ ) are Kazhdan-Lusztig polynomials of parabolic submodules of affine Hecke algebras of type $A$, so by [11], these polynomials are in $\mathbb{N}[q]$ (respectively $\mathbb{N}[p]$ ). Moreover, both canonical bases of $\mathbf{F}_{q}\left[\boldsymbol{s}_{l}\right]$ are dual to each other with respect to a certain bilinear form, which gives an inversion formula for Kazhdan-Lusztig polynomials; see [19, Thm. 5.15]. By [19], the basis $\left\{G^{+}\left(\lambda_{l}, s_{l}\right) \mid \lambda_{l} \in \Pi^{l}\right\}$ is a lower global crystal basis (in the sense of [8]) of the integrable $U_{q}\left(\widehat{\mathfrak{s l}}_{n}\right)$-module $\mathbf{F}_{q}\left[\boldsymbol{s}_{l}\right]$.

Let $\boldsymbol{s}_{n} \in \mathbb{Z}^{n}(s)$. In a similar way, one can define canonical bases $\left\{G^{\epsilon}\left(\lambda_{n}, \boldsymbol{s}_{n}\right)^{\bullet} \mid\right.$ $\left.\lambda_{n} \in \Pi^{n}\right\}(\epsilon= \pm 1)$ of the Fock space $\mathbf{F}_{p}\left[s_{n}\right]^{\bullet}$. By [19], the basis $\left\{G^{-}\left(\lambda_{n}, s_{n}\right)^{\bullet} \mid\right.$ $\left.\lambda_{n} \in \Pi^{n}\right\}$ is a lower global crystal basis of the integrable $U_{p}\left(\widehat{\mathfrak{s l}}_{l}\right)$-module $\mathbf{F}_{p}\left[\boldsymbol{s}_{n}\right]^{\bullet}$. For $\boldsymbol{\mu}_{n} \in \Pi^{n}, \epsilon= \pm 1$, write

$$
G^{\epsilon}\left(\boldsymbol{\mu}_{n}, \boldsymbol{s}_{n}\right)^{\bullet}=\sum_{\lambda_{n} \in \Pi^{n}} \dot{\Delta}_{\lambda_{n}, \boldsymbol{\mu}_{n} ; s_{n}}^{\epsilon}(q)\left|\lambda_{n}, \boldsymbol{s}_{n}\right\rangle^{\bullet},
$$

where the entries $\dot{\Delta}_{\lambda_{n}, \mu_{n} ; s_{n}}^{\epsilon}(q)$ are in $\mathbb{Z}\left[q, q^{-1}\right]$. Since the weight subspaces of $\Lambda^{s}$ are stable under the involution - , we have $\dot{\Delta}_{\lambda_{n}, \mu_{n} ; s_{n}}^{\epsilon}(q)=0$ unless $\operatorname{wt}\left(\left|\lambda_{n}, s_{n}\right\rangle^{\bullet}\right)=$ $\operatorname{wt}\left(\left|\boldsymbol{\mu}_{n}, \boldsymbol{s}_{n}\right\rangle^{\bullet}\right)$ and $\dot{\mathrm{wt}}\left(\left|\lambda_{n}, \boldsymbol{s}_{n}\right\rangle^{\bullet}\right)=\dot{\mathrm{wt}}\left(\left|\boldsymbol{\mu}_{n}, \boldsymbol{s}_{n}\right\rangle^{\bullet}\right)$. In this case, by Proposition 2.12 (ii), there exist $\boldsymbol{s}_{l} \in \mathbb{Z}^{l}(s)$ and $\lambda_{l}, \boldsymbol{\mu}_{l} \in \Pi^{l}$ such that $\left|\lambda_{l}, \boldsymbol{s}_{l}\right\rangle=\left|\lambda_{n}, \boldsymbol{s}_{n}\right\rangle^{\bullet}$ and $\left|\boldsymbol{\mu}_{l}, \boldsymbol{s}_{l}\right\rangle=$ $\left|\boldsymbol{\mu}_{n}, \boldsymbol{s}_{n}\right\rangle^{\bullet}$. It is then not hard to see that

$$
\dot{\Delta}_{\lambda_{n}, \boldsymbol{\mu}_{n} ; \boldsymbol{s}_{n}}^{\epsilon}(q)=\Delta_{\lambda_{l}, \boldsymbol{\mu}_{l} ; \boldsymbol{s}_{l}}^{\epsilon}(q) .
$$

3 Comparison of canonical bases of weight subspaces of $\mathrm{F}_{q}\left[s_{l}\right]\left(s_{l} \in \mathbb{Z}^{l}(s)\right)$

From now on, we shall use the following notation.

Notation 3.1 For $\boldsymbol{s}_{l} \in \mathbb{Z}^{l}(s)$ and $w \in \mathcal{P}\left(\mathbf{F}_{q}\left[\boldsymbol{s}_{l}\right]\right)$, put

$$
\left.\Pi^{l}\left(s_{l} ; w\right):=\left\{\lambda_{l} \in \Pi^{l}|| \lambda_{l}, s_{l}\right\rangle \in \Lambda^{s}\langle w\rangle\right\},
$$

and define similarly $\Pi^{n}\left(s_{n} ; \dot{w}\right)$ for $\boldsymbol{s}_{n} \in \mathbb{Z}^{n}(s)$ and $\dot{w} \in \dot{\mathcal{P}}\left(\mathbf{F}_{p}\left[s_{n}\right]^{\bullet}\right)$.

Definition 3.2 Let $\boldsymbol{s}_{l}, \boldsymbol{t}_{l} \in \mathbb{Z}^{l}(s), w \in \mathcal{P}\left(\mathbf{F}_{q}\left[\boldsymbol{s}_{l}\right]\right)$ and $w^{\prime} \in \mathcal{P}\left(\mathbf{F}_{q}\left[\boldsymbol{t}_{l}\right]\right)$. We say that the canonical bases of $\mathbf{F}_{q}\left[\boldsymbol{s}_{l}\right]\langle w\rangle$ and $\mathbf{F}_{q}\left[\boldsymbol{t}_{l}\right]\left\langle w^{\prime}\right\rangle$ are similar if there exists a bijection

$$
\sigma: \Pi^{l}\left(s_{l} ; w\right) \rightarrow \Pi^{l}\left(\boldsymbol{t}_{l} ; w^{\prime}\right)
$$

such that for all $\lambda_{l}, \boldsymbol{\mu}_{l} \in \Pi^{l}\left(s_{l} ; w\right), \epsilon= \pm 1$, we have

$$
\Delta_{\sigma\left(\lambda_{l}\right), \sigma\left(\boldsymbol{\mu}_{l}\right) ; \boldsymbol{t}_{l}}^{\epsilon}(q)=\Delta_{\lambda_{l}, \boldsymbol{\mu}_{l} ; \boldsymbol{s}_{l}}^{\epsilon}(q) .
$$

In other words, the canonical bases of $\mathbf{F}_{q}\left[\boldsymbol{s}_{l}\right]\langle w\rangle$ and $\mathbf{F}_{q}\left[\boldsymbol{t}_{l}\right]\left\langle w^{\prime}\right\rangle$ are similar if the transition matrices between the standard bases and the canonical bases are equal up to a reindexing of rows and columns. 
Notation 3.3 Throughout this section we fix a multi-charge $s_{l} \in \mathbb{Z}^{l}(s)$. To simplify, we drop the multi-charge $s_{l}$ in the notation of this section, that is we denote by $\lambda_{l}$ (respectively $G^{ \pm}\left(\lambda_{l}\right)$ ) the vector of the standard (respectively canonical) basis of $\mathbf{F}_{q}\left[s_{l}\right]$ indexed by the corresponding multi-partition and so on. In particular, we use the notation $\lambda_{l}$ either for a vector of the standard basis of $\mathbf{F}_{q}\left[\boldsymbol{s}_{l}\right]$ or for a vertex in the crystal graph $\mathcal{B}:=\mathcal{B}\left[s_{l}\right]$.

\subsection{The bijections $\sigma_{i}$}

We recall here the definition of the involution $\sigma_{i}(0 \leq i \leq n-1)$ of the crystal graph $\mathcal{B}$. We sometimes view $\sigma_{i}$ as a bijection of $\Pi^{l}$.

Definition 3.4 Let $\lambda_{l} \in \mathcal{B} \cong \Pi^{l}$ and $i \in \llbracket 0 ; n-1 \rrbracket$. Let $\mathcal{C}$ be the $i$-chain in $\mathcal{B}$ containing $\lambda_{l}$. Let $\sigma_{i}\left(\boldsymbol{\lambda}_{l}\right) \in \mathcal{B} \cong \Pi^{l}$ be the unique element in $\mathcal{C}$ such that $\operatorname{wt}\left(\sigma_{i}\left(\boldsymbol{\lambda}_{l}\right)\right)=$ $\sigma_{i} \cdot\left(\operatorname{wt}\left(\boldsymbol{\lambda}_{l}\right)\right)$. In other words, $\sigma_{i}\left(\boldsymbol{\lambda}_{l}\right)$ is obtained from $\boldsymbol{\lambda}_{l}$ via a central symmetry in the middle of $\mathcal{C}$. This defines an involution $\sigma_{i}$ of $\mathcal{B}$. This map induces, for $w \in \mathcal{P}\left(\mathbf{F}_{q}\left[\boldsymbol{s}_{l}\right]\right)$, a bijection

$$
\sigma_{i}: \Pi^{l}\left(s_{l} ; w\right) \stackrel{\sim}{\longrightarrow} \Pi^{l}\left(s_{l} ; \sigma_{i} . w\right)
$$

By [9], the definition of $\sigma_{0}, \ldots, \sigma_{n-1}$ as bijections of $\mathcal{B}$ gives actually rise to an action of the Weyl group $W_{n}$ on $\mathcal{B}$, but we do not need this fact in this article.

Proposition 3.5 Let $w \in \mathcal{P}\left(\mathbf{F}_{q}\left[s_{l}\right]\right)$ and $i \in \llbracket 0 ; n-1 \rrbracket$ be such that $w+\alpha_{i} \notin$ $\mathcal{P}\left(\mathbf{F}_{q}\left[\boldsymbol{s}_{l}\right]\right)$. Let $\lambda_{l} \in \Pi^{l}\left(\boldsymbol{s}_{l} ; w\right)$ and $\boldsymbol{\mu}_{l}:=\sigma_{i}\left(\boldsymbol{\lambda}_{l}\right)$. Then we have the following:

(i) $\mu_{l}$ is the multi-partition obtained by adding to $\lambda_{l}$ all its addable $i$-nodes, and we have $\left|\boldsymbol{\mu}_{l} \backslash \lambda_{l}\right|=k_{i}:=\left(w, \alpha_{i}\right)$.

(ii) In $\mathbf{F}_{q}\left[\boldsymbol{s}_{l}\right]$, we have $\boldsymbol{\mu}_{l}=f_{i}^{\left(k_{i}\right)} \cdot \lambda_{l}$ and $\lambda_{l}=e_{i}^{\left(k_{i}\right)} \cdot \boldsymbol{\mu}_{l}$.

(iii) In $\mathcal{B}$, we have $\boldsymbol{\mu}_{l}=\left(\tilde{f}_{i}^{\text {low }}\right)^{k_{i}} \cdot \lambda_{l}$ and $\lambda_{l}=\left(\tilde{e}_{i}^{\text {low }}\right)^{k_{i}} \cdot \mu_{l}$.

Proof Let $\mathcal{C}$ be the $i$-chain in $\mathcal{B}$ containing $\lambda_{l}$ and $\boldsymbol{\mu}_{l}$. Since $w+\alpha_{i}$ is not a weight of $\mathbf{F}_{q}\left[s_{l}\right], \lambda_{l}$ has no removable $i$-node. This implies, by [19, Thm. 2.4], that $\lambda_{l}$ is the head of the chain $\mathcal{C}$, and by symmetry $\boldsymbol{\mu}_{l}$ is the tail of $\mathcal{C}$. Note that since $w+\alpha_{i}$ is not a weight of $\mathbf{F}_{q}\left[\boldsymbol{s}_{l}\right], \sigma_{i} .\left(w+\alpha_{i}\right)=\left(\sigma_{i} . w\right)-\alpha_{i}$ is also not a weight of $\mathbf{F}_{q}\left[\boldsymbol{s}_{l}\right]$, so $\boldsymbol{\mu}_{l}$ has no addable $i$-node. By [19, Thm. 2.4], $\boldsymbol{\mu}_{l}$ is obtained by adding some $i$-nodes (let us say $k$ of them) to $\lambda_{l}$. The integer $k=\left|\boldsymbol{\mu}_{l} \backslash \lambda_{l}\right|$ is none other than the length of the chain $\mathcal{C}$. By a well-known formula for crystal graphs relating weights and positions in the $i$-chains, since $\lambda_{l}$ is the head of $\mathcal{C}$, we have $k_{i}=\left(\operatorname{wt}\left(\lambda_{l}\right), \alpha_{i}\right)=k$. Thus $\mathcal{C}$ is a $i$-chain of length $k_{i}$ with head $\lambda_{l}$ and tail $\boldsymbol{\mu}_{l}$, which proves (iii). The divided powers $e_{i}^{(k)}, f_{i}^{(k)} \in U_{q}\left(\widehat{\mathfrak{s l}}_{n}\right)\left(k \in \mathbb{N}^{*}\right)$ act on $\boldsymbol{v}_{l} \in \mathbf{F}_{q}\left[s_{l}\right]$ as follows, with notation of Section 2.2.2:

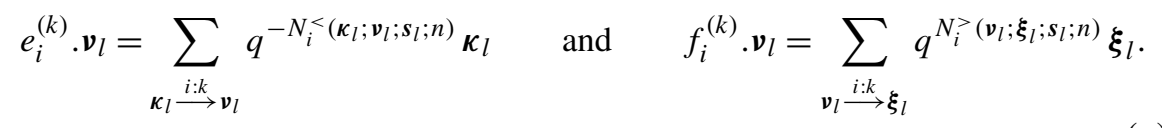


(For $k=1$, this is a part of Theorem 2.3. The general case follows by induction on $k$; see e.g. [5] for a detailed proof.) Since $\mu_{l}$ has no addable $i$-node and $\lambda_{l}$ has no removable $i$-node, $\boldsymbol{\mu}_{l} \backslash \lambda_{l}$ is the set of the addable $i$-nodes of $\lambda_{l}$ and also the set of the removable $i$-nodes of $\boldsymbol{\mu}_{l}$, and this set has exactly $k_{i}$ elements. This proves (i), and this together with $(*)$ proves (ii).

Example 3.6 Take $n=3, l=2, s_{l}=(1,2), i=0, \lambda_{l}=((2,2,1),(3,2))$ and $w=$ $\operatorname{wt}\left(\left|\lambda_{l}, s_{l}\right\rangle\right)$. Then we have $\sigma_{i}\left(\lambda_{l}\right)=((3,2,2),(3,3,1))$. Note that $w+\alpha_{i}$ is not a weight of $\mathbf{F}_{q}\left[\boldsymbol{s}_{l}\right]$, so Proposition 3.5 can be applied in this case.

Remark 3.7 The proof of Proposition 3.5 shows (with the assumptions and notation of this proposition) that $\lambda_{l}=\sigma_{i}^{-1}\left(\mu_{l}\right)$ is the multi-partition obtained by removing to $\boldsymbol{\mu}_{l}$ all its removable $i$-nodes.

3.2 A first theorem of comparison

Define a symmetric bilinear non-degenerate form $(.,$.$) on \mathbf{F}_{q}\left[\boldsymbol{s}_{l}\right]$ by

$$
\left(\lambda_{l}, \mu_{l}\right)=q^{\left\|\lambda_{l}\right\|} \delta_{\lambda_{l}, \mu_{l}} \quad\left(\lambda_{l}, \mu_{l} \in \Pi^{l}\right),
$$

where we put

$$
\left\|\lambda_{l}\right\|:=\left(\operatorname{wt}\left(\lambda_{l}\right), \operatorname{wt}\left(\lambda_{l}\right)\right) / 2 \quad\left(\lambda_{l} \in \Pi^{l}\right) .
$$

This form enjoys the following property:

Lemma 3.8 For $u, v \in \mathbf{F}_{q}\left[s_{l}\right], 0 \leq i \leq n-1$, we have

$$
\left(t_{i} \cdot u, v\right)=\left(u, t_{i} \cdot v\right) \quad \text { and } \quad\left(e_{i} \cdot u, v\right)=\left(u, f_{i} \cdot v\right) .
$$

Proof Identical to the proof of [12, Prop. 8.1].

Let $\left\{G^{*}\left(\lambda_{l}\right) \mid \lambda_{l} \in \Pi^{l}\right\}$ denote the adjoint basis of $\left\{G^{+}\left(\lambda_{l}\right) \mid \lambda_{l} \in \Pi^{l}\right\}$ with respect to the form (.,.). Since the basis $\left\{G^{+}\left(\lambda_{l}\right) \mid \lambda_{l} \in \Pi^{l}\right\}$ is a lower global crystal basis of $\mathbf{F}_{q}\left[s_{l}\right]$ in the sense of [8], it follows by Lemma 3.8 and [8, Prop. 3.2.2] that $\left\{G^{*}\left(\lambda_{l}\right) \mid \lambda_{l} \in \Pi^{l}\right\}$ is an upper global crystal basis of $\mathbf{F}_{q}\left[s_{l}\right]$.

We are now ready to prove the following result, which is a generalization to higherlevel Fock spaces of [13, Thm. 20].

Theorem 3.9 Let $\boldsymbol{s}_{l} \in \mathbb{Z}^{l}(s), w \in \mathcal{P}\left(\mathbf{F}_{q}\left[s_{l}\right]\right)$ and $i \in \llbracket 0 ; n-1 \rrbracket$ be such that $w+\alpha_{i}$ is not a weight of $\mathbf{F}_{q}\left[\boldsymbol{s}_{l}\right]$. Let $\sigma_{i}: \Pi^{l}\left(\boldsymbol{s}_{l} ; w\right) \rightarrow \Pi^{l}\left(\boldsymbol{s}_{l} ; \sigma_{i} . w\right)$ be the bijection defined by (54). Then we have, for $\lambda_{l}, \boldsymbol{\mu}_{l} \in \Pi^{l}\left(\boldsymbol{s}_{l} ; w\right)$,

$$
\begin{aligned}
& \text { (i) } \Delta_{\sigma_{i}\left(\lambda_{l}\right), \sigma_{i}\left(\boldsymbol{\mu}_{l}\right) ; s_{l}}^{+}(q)=\Delta_{\lambda_{l}, \mu_{l} ; s_{l}}^{+}(q) \text { and } \\
& \text { (ii) } \Delta_{\sigma_{i}\left(\lambda_{l}\right), \sigma_{i}\left(\mu_{l}\right) ; s_{l}}^{-}(q)=\Delta_{\lambda_{l}, \mu_{l} ; s_{l}}^{-}(q) \text {. }
\end{aligned}
$$


As a consequence, the canonical bases of $\mathbf{F}_{q}\left[\boldsymbol{s}_{l}\right]\langle w\rangle$ and $\mathbf{F}_{q}\left[\boldsymbol{s}_{l}\right]\left\langle\sigma_{i} . w\right\rangle$ are similar in the sense of Definition 3.2.

Proof Let us prove (i). Let $\boldsymbol{\mu}_{l} \in \Pi^{l}\left(s_{l} ; w\right)$. Taking adjoint bases in (49) yields

$$
q^{-\left\|\sigma_{i}\left(\boldsymbol{\mu}_{l}\right)\right\|} \sigma_{i}\left(\boldsymbol{\mu}_{l}\right)=\sum_{\boldsymbol{v}_{l} \in \Pi^{l}\left(\boldsymbol{s}_{l} ; \sigma_{i}, w\right)} \Delta_{\sigma_{i}\left(\boldsymbol{\mu}_{l}\right), \boldsymbol{v}_{l} ; \boldsymbol{s}_{l}}^{+}(q) G^{*}\left(\boldsymbol{v}_{l}\right) .
$$

Since $\sigma_{i}: \Pi^{l}\left(s_{l} ; w\right) \rightarrow \Pi^{l}\left(s_{l} ; \sigma_{i} . w\right)$ is a bijection, we can make in the sum above the reindexing $\boldsymbol{v}_{l}=\sigma_{i}\left(\lambda_{l}\right)$. If we now apply $e_{i}^{\left(k_{i}\right)}$ with $k_{i}:=\left(w, \alpha_{i}\right)$ to both hand-sides of this equality, we get

$$
q^{-\left\|\sigma_{i}\left(\boldsymbol{\mu}_{l}\right)\right\|} e_{i}^{\left(k_{i}\right)} . \sigma_{i}\left(\boldsymbol{\mu}_{l}\right)=\sum_{\lambda_{l} \in \Pi^{l}\left(\boldsymbol{s}_{l} ; w\right)} \Delta_{\sigma_{i}\left(\boldsymbol{\mu}_{l}\right), \sigma_{i}\left(\boldsymbol{\lambda}_{l}\right) ; s_{l}}^{+}(q) e_{i}^{\left(k_{i}\right)} \cdot G^{*}\left(\sigma_{i}\left(\boldsymbol{\lambda}_{l}\right)\right) .
$$

Note that

$$
\left\|\sigma_{i}\left(\boldsymbol{\mu}_{l}\right)\right\|=\left(\sigma_{i} \cdot \operatorname{wt}\left(\boldsymbol{\mu}_{l}\right), \sigma_{i} \cdot \operatorname{wt}\left(\boldsymbol{\mu}_{l}\right)\right) / 2=\left(\operatorname{wt}\left(\boldsymbol{\mu}_{l}\right), \operatorname{wt}\left(\boldsymbol{\mu}_{l}\right)\right) / 2=\left\|\boldsymbol{\mu}_{l}\right\| .
$$

By Proposition 3.5 (ii), we have $e_{i}^{\left(k_{i}\right)} . \sigma_{i}\left(\boldsymbol{\mu}_{l}\right)=\boldsymbol{\mu}_{l}$. Now let $\lambda_{l} \in \Pi^{l}\left(\boldsymbol{s}_{l} ; w\right)$. Since $w+\alpha_{i}$ is not a weight of $\mathbf{F}_{q}\left[s_{l}\right]$, we have $e_{i} \cdot \lambda_{l}=0$, whence $\tilde{e}_{i}{ }^{\text {up }} \cdot \lambda_{l}=0$. Moreover, again by Proposition 3.5, we have $\sigma_{i}\left(\lambda_{l}\right)=f_{i}^{\left(k_{i}\right)} . \lambda_{l}$. Therefore, by Lemma 2.15, we have

$$
\lambda_{l}=\left({\tilde{e_{i}}}^{\text {low }}\right)^{k_{i}} \cdot\left(f_{i}^{\left(k_{i}\right)} \cdot \lambda_{l}\right)=\left(\tilde{e}_{i}^{\text {up }}\right)^{k_{i}} \cdot\left(f_{i}^{\left(k_{i}\right)} \cdot \lambda_{l}\right)=\left(\tilde{e}_{i}^{\text {up }}\right)^{k_{i}} \cdot\left(\sigma_{i}\left(\lambda_{l}\right)\right),
$$

whence $\left(\tilde{e}_{i}^{\text {up }}\right)^{k_{i}+1} \cdot \sigma_{i}\left(\boldsymbol{\lambda}_{l}\right)=0$. Since $\left\{G^{*}\left(\boldsymbol{v}_{l}\right) \mid \boldsymbol{v}_{l} \in \Pi^{l}\right\}$ is an upper global crystal basis of $\mathbf{F}_{q}\left[s_{l}\right]$, [8, Lemma 5.1.1 (ii)] then implies

$$
e_{i}^{\left(k_{i}\right)} \cdot G^{*}\left(\sigma_{i}\left(\lambda_{l}\right)\right)=G^{*}\left(\left(\tilde{e}_{i}^{\text {up }}\right)^{k_{i}} \cdot\left(\sigma_{i}\left(\lambda_{l}\right)\right)\right)=G^{*}\left(\lambda_{l}\right) . \quad(* * *)
$$

Combining $(*),(* *)$ and $(* * *)$ we get

$$
q^{-\left\|\boldsymbol{\mu}_{l}\right\|} \boldsymbol{\mu}_{l}=\sum_{\lambda_{l} \in \Pi^{l}\left(\boldsymbol{s}_{l} ; w\right)} \Delta_{\sigma_{i}\left(\boldsymbol{\mu}_{l}\right), \sigma_{i}\left(\boldsymbol{\lambda}_{l}\right) ; \boldsymbol{s}_{l}}^{+}(q) G^{*}\left(\boldsymbol{\lambda}_{l}\right) .
$$

Since this is valid for any $\boldsymbol{\mu}_{l} \in \Pi^{l}\left(s_{l} ; w\right)$, we get the claimed formula by taking again adjoint bases.

Let us now prove (ii). Let $w^{\prime} \in \mathcal{P}\left(\mathbf{F}_{q}\left[\boldsymbol{s}_{l}\right]\right)$. If we know the basis $\left\{G^{+}\left(\lambda_{l}\right) \mid \lambda_{l} \in\right.$ $\left.\Pi^{l}\left(s_{l} ; w^{\prime}\right)\right\}$, then we can compute the involution of $\mathbf{F}_{q}\left[s_{l}\right]\left\langle w^{\prime}\right\rangle$ by solving a unitriangular system. Since the canonical basis $\left\{G^{-}\left(\lambda_{l}\right) \mid \lambda_{l} \in \Pi^{l}\left(s_{l} ; w^{\prime}\right)\right\}$ is uniquely determined by the involution of $\mathbf{F}_{q}\left[s_{l}\right]\left\langle w^{\prime}\right\rangle$, the basis $\left\{G^{-}\left(\lambda_{l}\right) \mid \lambda_{l} \in \Pi^{l}\left(s_{l} ; w^{\prime}\right)\right\}$ is uniquely determined by the basis $\left\{G^{+}\left(\lambda_{l}\right) \mid \lambda_{l} \in \Pi^{l}\left(s_{l} ; w^{\prime}\right)\right\}$ (and conversely). Thus (i) implies (ii). (For a different proof of this fact, one can also apply [19, Thm 5.15].)

Example 3.10 Take $n=3, l=2, \boldsymbol{s}_{l}=(1,0), w=\mathrm{wt}\left(\left|\emptyset_{l}, \boldsymbol{s}_{l}\right\rangle\right)-\left(2 \alpha_{0}+3 \alpha_{1}+\alpha_{2}\right)$ and $i=2$. One can easily check that $w+\alpha_{i}$ is not a weight of $\mathbf{F}_{q}\left[\boldsymbol{s}_{l}\right]$. The elements 
of $\Pi^{l}\left(s_{l} ; w\right)$ are

$$
\begin{aligned}
& ((1),(5)),((4),(2)),((4,2), \varnothing),((1),(2,2,1)),((2,2),(2)), \\
& ((1,1),(2,1,1)),((1,1,1,1),(2)),((1),(2,1,1,1)),
\end{aligned}
$$

and their respective images by the map $\sigma_{i}$ are

$$
\begin{aligned}
& ((2),(6,1)),((5),(3,1)),((5,3,1), \varnothing),((2),(3,2,2)),((2,2,1),(3,1)), \\
& ((2,1,1),(3,1,1)),((2,1,1,1),(3,1)),((2),(3,1,1,1,1)) .
\end{aligned}
$$

With obvious notation, the transition matrices of the canonical bases of $\mathbf{F}_{q}\left[\boldsymbol{s}_{l}\right]\langle w\rangle$ are

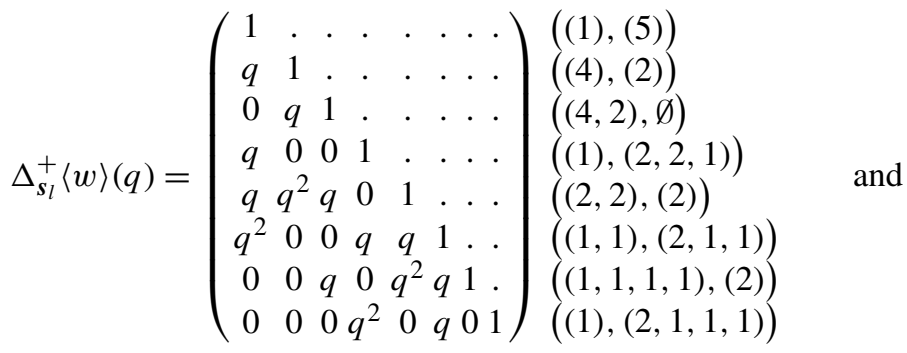

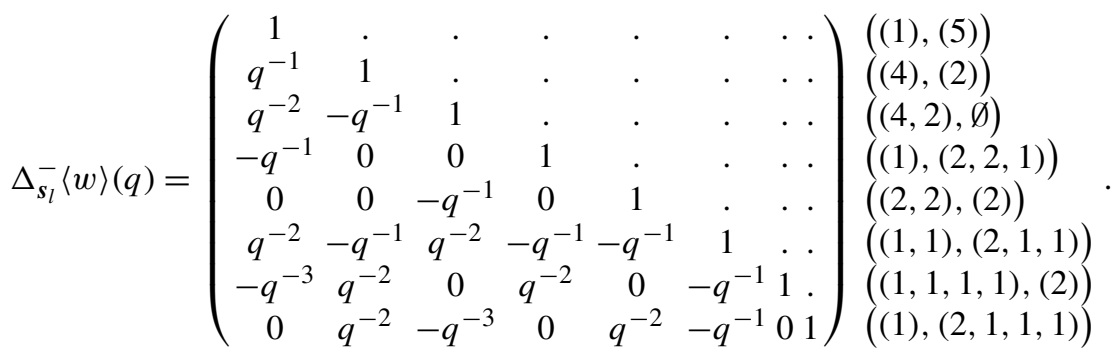

In the same way, the transition matrices of the canonical bases of $\mathbf{F}_{q}\left[\boldsymbol{s}_{l}\right]\left\langle\sigma_{i} . w\right\rangle$ are

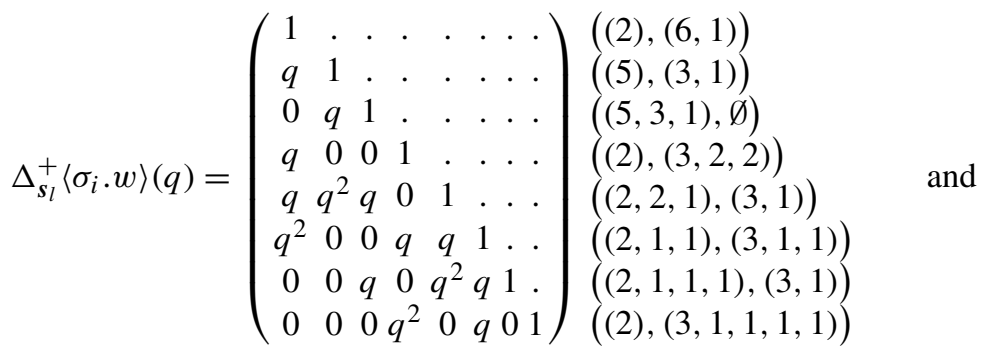




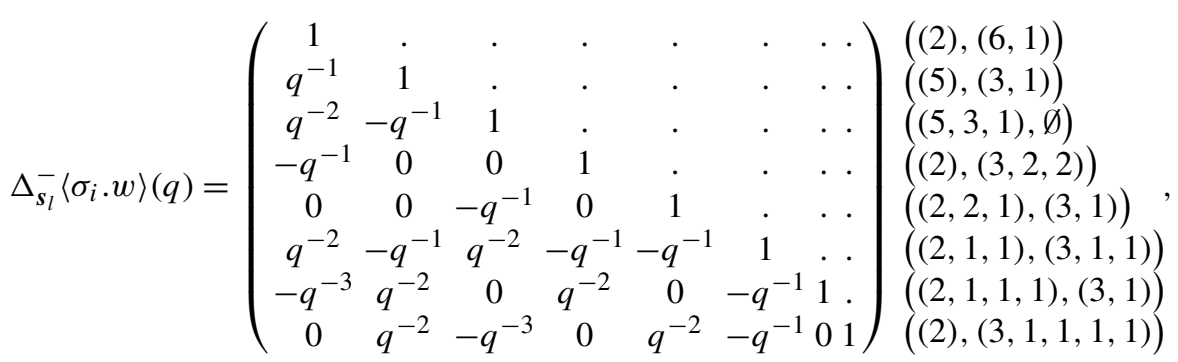

in agreement with Theorem 3.9.

\section{Comparison of canonical bases of $\mathrm{F}_{q}\left[s_{l}\right]\langle w\rangle$ for $w$ in a given coset in $\mathcal{P}\left(\Lambda^{s}\right) / \mathbb{Z} \delta$ and different multi-charges $s_{l}$}

Notation 4.1 For $\boldsymbol{a}_{l}, \boldsymbol{b}_{l} \in \mathbb{Z}^{l}(s)$, introduce the shorter notation

$$
d\left(\boldsymbol{a}_{l}, \boldsymbol{b}_{l}\right):=\Delta\left(\boldsymbol{a}_{l}, n\right)-\Delta\left(\boldsymbol{b}_{l}, n\right) \in \mathbb{Z} .
$$

Throughout this section we fix $s_{l} \in \mathbb{Z}^{l}(s), w \in \mathcal{P}\left(\mathbf{F}_{q}\left[\boldsymbol{s}_{l}\right]\right)$ and $i \in \llbracket 0 ; l-1 \rrbracket$. Finally, let $\left(s_{n}, \dot{w}\right) \in \mathbb{Z}^{n}(s) \times \dot{\mathcal{P}}\left(\Lambda^{s}\right)$ be the pair such that $\mathbf{F}_{q}\left[s_{l}\right]\langle w\rangle=\mathbf{F}_{p}\left[s_{n}\right]^{\bullet}\langle\dot{w}\rangle($ see Proposition 2.12 (i)).

\subsection{A second theorem of comparison}

Recall that $\dot{W}_{l}=\left\langle\dot{\sigma}_{0}, \ldots, \dot{\sigma}_{l-1}\right\rangle \cong \widetilde{\mathfrak{S}}_{l}$ is the Weyl group of $U_{p}\left(\widehat{\mathfrak{s l}}_{l}\right)$.

Definition 4.2 Keep Notation 4.1. By analogy with Definition 3.4, define a bijection

$$
\dot{\sigma}_{i}: \Pi^{n}\left(s_{n} ; \dot{w}\right) \rightarrow \Pi^{n}\left(s_{n} ; \dot{\sigma}_{i} \cdot \dot{w}\right)
$$

which enjoys similar properties as the bijections $\sigma_{j}$ from (54). Since $\mathbf{F}_{q}\left[\boldsymbol{s}_{l}\right]\langle w\rangle=$ $\mathbf{F}_{p}\left[\boldsymbol{s}_{n}\right]^{\bullet}\langle\dot{w}\rangle$, we have a bijection between the standard basis of $\mathbf{F}_{q}\left[\boldsymbol{s}_{l}\right]\langle w\rangle$ (as a subspace of $\mathbf{F}_{q}\left[\boldsymbol{s}_{l}\right]$ ) and the standard basis of $\mathbf{F}_{p}\left[\boldsymbol{s}_{n}\right]^{\bullet}\langle\dot{w}\rangle$ (as a subspace of $\mathbf{F}_{p}\left[\boldsymbol{s}_{n}\right]^{\bullet}$ ). We thus have a bijection $\Pi^{l}\left(s_{l} ; w\right) \stackrel{\sim}{\longrightarrow} \Pi^{n}\left(s_{n} ; \dot{w}\right)$. Put $\boldsymbol{t}_{l}:=\dot{\sigma}_{i} \cdot \boldsymbol{s}_{l}$. The same argument gives, by Lemma 2.14 , a bijection $\Pi^{\mid}\left(\boldsymbol{t}_{\mid} ; w+d\left(\boldsymbol{s}_{\mid}, \boldsymbol{t}_{\mid}\right) \delta\right) \stackrel{\sim}{\longrightarrow} \Pi^{n}\left(\boldsymbol{s}_{n} ; \dot{\sigma}_{i} . \dot{w}\right)$. We therefore have the following commutative diagram of bijections, in which the dashed arrow will still be denoted by $\dot{\sigma}_{i}$.

$$
\begin{aligned}
& \Pi_{\uparrow}^{n}\left(\boldsymbol{s}_{n} ; \dot{w}\right) \stackrel{\dot{\sigma}_{i}}{\longrightarrow} \underset{\uparrow}{\longrightarrow} \quad \Pi^{n}\left(\boldsymbol{s}_{n} ; \dot{\sigma}_{i} . \dot{w}\right) \\
& \Pi^{l}\left(s_{l} ; w\right) \stackrel{\sim}{\sim} \underset{\sim}{\sim} \Pi^{l}\left(\dot{\sigma}_{i} . s_{l} ; w+d\left(s_{l}, \dot{\sigma}_{i} . s_{l}\right) \delta\right) .
\end{aligned}
$$

Example 4.3 Take $n=2, l=3, \boldsymbol{s}_{l}=(0,2,-1)$ and $i=2$. Note that $d\left(\boldsymbol{s}_{l}, \dot{\sigma}_{i} . \boldsymbol{s}_{l}\right)=0$ in this case. Take $\lambda_{l}=(\emptyset,(2), \emptyset) \in \Pi^{l}\left(s_{l} ; w\right)$, where $w:=\operatorname{wt}\left(\left|\emptyset_{l}, s_{l}\right\rangle\right)-\left(\alpha_{0}+\alpha_{1}\right)$. By Proposition 2.12 (i), we have $\dot{w}=\dot{w} t\left(\left|\emptyset_{n}, s_{n}\right\rangle^{\bullet}\right)-\left(2 \dot{\alpha}_{0}+3 \dot{\alpha}_{1}+\dot{\alpha}_{2}\right)$ with $s_{n}:=(1,0)$. We have $\left|\lambda_{l}, s_{l}\right\rangle=\left|\lambda_{n}, s_{n}\right\rangle^{\bullet}$ with $\lambda_{n}:=((1),(5))$. One computes 
$\boldsymbol{\mu}_{n}:=\dot{\sigma}_{i}\left(\boldsymbol{\lambda}_{n}\right)=((2),(6,1))$ (see Example 3.10). Let $\boldsymbol{t}_{l}:=\dot{\sigma}_{i} . \boldsymbol{s}_{l}=(0,-1,2)$. Then $\dot{\sigma}_{i}\left(\lambda_{l}\right)$ is the $l$-multi-partition such that $\left|\dot{\sigma}_{i}\left(\lambda_{l}\right), \boldsymbol{t}_{l}\right\rangle=\left|\boldsymbol{\mu}_{n}, \boldsymbol{s}_{n}\right\rangle^{\bullet}$, namely $\dot{\sigma}_{i}\left(\boldsymbol{\lambda}_{l}\right)=$ $(\emptyset, \emptyset,(2)) . \diamond$

Since $\left\{G^{-}\left(\boldsymbol{\lambda}_{n}, \boldsymbol{s}_{n}\right)^{\bullet} \mid \lambda_{n} \in \Pi^{n}\right\}$ is a lower global crystal basis of $\mathbf{F}_{p}\left[\boldsymbol{s}_{n}\right]^{\bullet}$, one can prove for the Fock space $\mathbf{F}_{p}\left[\boldsymbol{s}_{n}\right]^{\bullet}$ an analogue of Theorem 3.9. Rephrasing this result in terms of the indexation $\lambda_{l}$ leads to the following result.

Theorem 4.4 Keep Notation 4.1 and assume that $\dot{w}+\dot{\alpha}_{i}$ is not a weight of $\mathbf{F}_{p}\left[\boldsymbol{s}_{n}\right]^{\bullet}$. Let $\dot{\sigma}_{i}: \Pi^{l}\left(s_{l} ; w\right) \rightarrow \Pi^{l}\left(\dot{\sigma}_{i} . s_{l} ; w+d\left(s_{l}, \dot{\sigma}_{i} . s_{l}\right) \delta\right)$ be the bijection from Definition 4.2. Then we have, for $\lambda_{l}, \mu_{l} \in \Pi^{l}\left(s_{l} ; w\right), \epsilon= \pm 1$ :

$$
\Delta_{\dot{\sigma}_{i}\left(\lambda_{l}\right), \dot{\sigma}_{i}\left(\mu_{l}\right) ; \dot{\sigma}_{i} . s_{l}}^{\epsilon}(q)=\Delta_{\lambda_{l}, \mu_{l} ; s_{l}}^{\epsilon}(q)
$$

Proof Apply the analogue for $\mathbf{F}_{p}\left[\boldsymbol{s}_{n}\right]^{\bullet}$ of Theorem 3.9 mentioned above, then use Lemma 2.14 and (52).

Example 4.5 (see Examples 3.10 and 4.3) Take $n, l, s_{l}, w$ and $i$ as in Example 4.3 (namely, $n:=2, l:=3, s_{l}:=(0,2,-1), w:=\operatorname{wt}\left(\left|\emptyset_{l}, s_{l}\right\rangle\right)-\left(\alpha_{0}+\alpha_{1}\right)$ and $i:=$ 2). Note that $\dot{w}+\dot{\alpha}_{i} \notin \dot{\mathcal{P}}\left(\mathbf{F}_{p}\left[s_{n}\right]^{\bullet}\right)$ (see Example 3.10). The elements of $\Pi^{l}\left(s_{l} ; w\right)$ are

$$
\begin{aligned}
& (\emptyset,(2), \varnothing),(\varnothing, \emptyset,(1,1)),(\varnothing,(1),(1)),(\emptyset, \emptyset,(2)), \\
& ((2), \varnothing, \varnothing),((1), \varnothing,(1)),((1,1), \varnothing, \emptyset),(\emptyset, \varnothing,(1,1)),
\end{aligned}
$$

and their respective images by the map $\dot{\sigma}_{i}$ are

$$
\begin{aligned}
& (\emptyset, \emptyset,(2)),(\varnothing, \emptyset,(1,1)),(\varnothing,(1),(1)),((2), \varnothing, \emptyset), \\
& (\emptyset,(2), \varnothing),((1),(1), \varnothing),((1,1), \varnothing, \varnothing),(\varnothing,(1,1), \varnothing),
\end{aligned}
$$

On the one hand, the transition matrices of the canonical bases of $\mathbf{F}_{q}\left[\boldsymbol{s}_{l}\right]\langle w\rangle$ are

$$
\Delta_{\boldsymbol{s}_{l}}^{+}\langle w\rangle(q)=\left(\begin{array}{ccccccc|l}
1 & . & . & . & . & . & . & (\emptyset,(2), \emptyset) \\
q & 1 & . & . & . & . & . & (\emptyset, \emptyset,(1,1)) \\
q^{2} & q & 1 & . & . & . & . & (\emptyset,(1),(1)) \\
0 & 0 & q & 1 & . & . & . & (\emptyset, \emptyset,(2)) \\
q & 0 & 0 & 0 & 1 & . & . & ((2), \emptyset, \emptyset) \\
q^{2} & q & q^{2} & q & q & 1 & . & (1), \emptyset,(1)) \\
q^{3} & q^{2} & 0 & 0 & q^{2} & q & 1 \\
0 & q^{2} & q^{3} & q^{2} & 0 & q & 0 & 1
\end{array}\right)\left(\begin{array}{l}
(1,1), \emptyset, \emptyset) \\
(\emptyset, \emptyset,(1,1))
\end{array}\right. \text { and }
$$




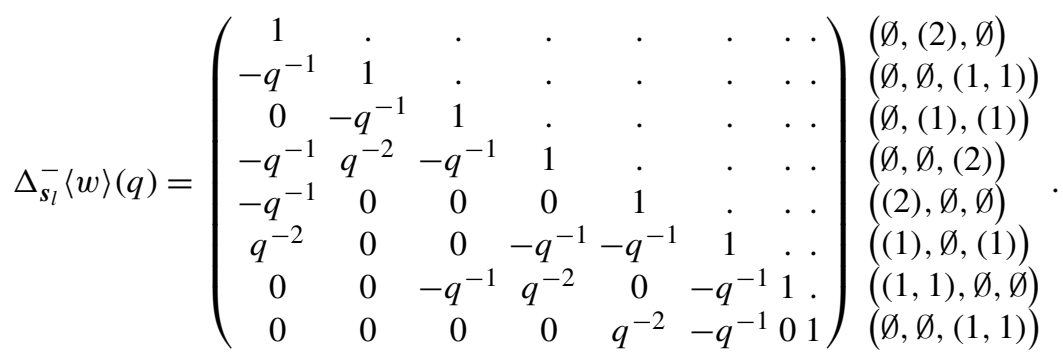

On the other hand, the transition matrices of the canonical bases of $\mathbf{F}_{q}\left[\dot{\sigma}_{i} . \boldsymbol{s}_{l}\right]\langle w\rangle$ are

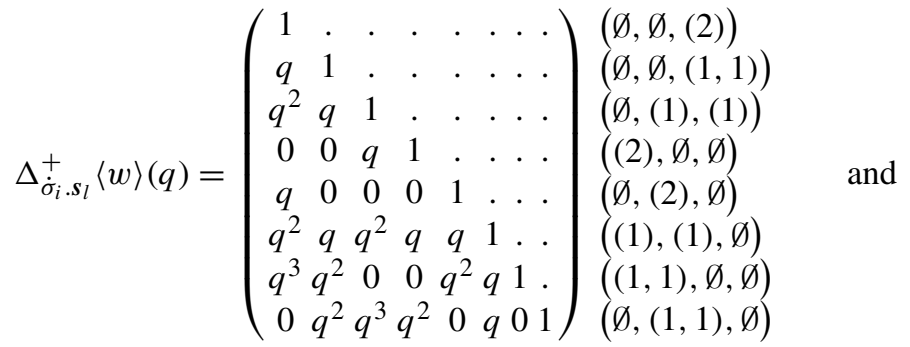

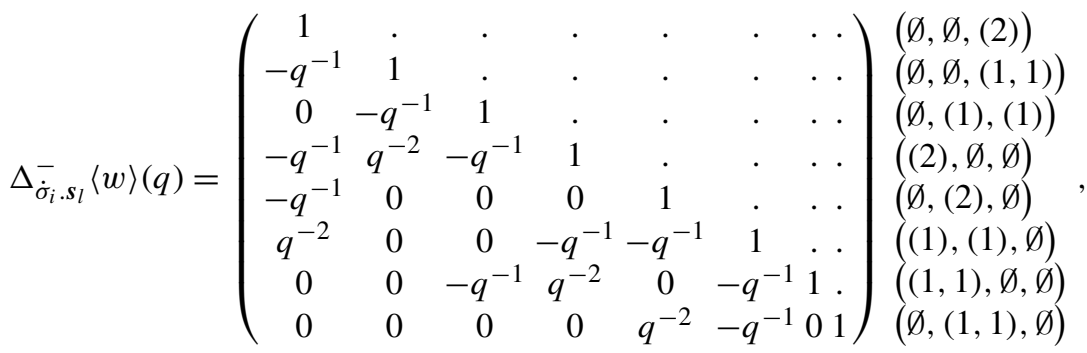

in agreement with Theorem 4.4 (note that $d\left(s_{l}, \dot{\sigma}_{i} . s_{l}\right)=0$ in this case).

\subsection{A sufficient condition for Theorem 4.4}

Keep again Notation 4.1. We can apply Theorem 4.4 if $\dot{w}+\dot{\alpha}_{i}$ is not a weight of $\mathbf{F}_{p}\left[\boldsymbol{s}_{n}\right]^{\bullet}$. By [7, Prop. 3.6 (iv)], this condition holds if and only if $\dot{e}_{i} .\left(\left|\lambda_{l}, s_{l}\right\rangle\right)=0$ for all $\lambda_{l} \in \Pi^{l}\left(s_{l} ; w\right)$. We therefore have to check, for all $\lambda_{l} \in \Pi^{l}\left(s_{l} ; w\right)$, whether $\lambda_{n}$ has a removable $i$-node, where $\lambda_{n} \in \Pi^{n}$ is related to $\lambda_{l}$ by $\left|\lambda_{l}, s_{l}\right\rangle=\left|\lambda_{n}, s_{n}\right\rangle^{\bullet}$. It is not very convenient to make such tests in practice when the cardinality of $\Pi^{l}\left(s_{l} ; w\right)$ is large. We shall therefore give a sufficient condition on $s_{l}$ and $w$ that ensures, without further computation, that $\dot{w}+\dot{\alpha}_{i}$ is not a weight of $\mathbf{F}_{p}\left[\boldsymbol{s}_{n}\right]^{\bullet}$.

Notation 4.6 Let $\boldsymbol{s}_{l} \in \mathbb{Z}^{l}(s), w \in \mathcal{P}\left(\mathbf{F}_{q}\left[\boldsymbol{s}_{l}\right]\right)$ and $i \in \llbracket 0 ; l-1 \rrbracket$. By (32), the integer $M_{i}\left(\lambda_{l} ; s_{l} ; n\right)$ only depends on $s_{l}$ and $w$, but not on $\lambda_{l} \in \Pi^{l}\left(s_{l} ; w\right)$. From now on, this number will be denoted by $M_{i}\left(w ; s_{l}\right)$. 
Lemma 4.7 Keep Notation 4.1. Then for all $\dot{\sigma} \in \dot{W}_{l}, 0 \leq i \leq n-1, w \in \mathcal{P}\left(\mathbf{F}_{q}\left[s_{l}\right]\right)$, we have

$$
M_{i}\left(w+d\left(s_{l}, \dot{\sigma} \cdot s_{l}\right) \delta ; \dot{\sigma} \cdot s_{l}\right)=M_{i}\left(w ; s_{l}\right)
$$

Proof By (32) and the definition of the integers $M_{j}\left(w+d\left(s_{l}, \dot{\sigma} . s_{l}\right) \delta ; \dot{\sigma} . s_{l}\right)$ and $d\left(s_{l}, \dot{\sigma} . s_{l}\right)$, we have

$$
\begin{aligned}
w & +d\left(s_{l}, \dot{\sigma} \cdot s_{l}\right) \delta \\
& =\operatorname{wt}\left(\left|\emptyset_{l}, \dot{\sigma} \cdot s_{l}\right\rangle\right)-\sum_{j=0}^{n-1} M_{j}\left(w+d\left(s_{l}, \dot{\sigma} . s_{l}\right) \delta ; \dot{\sigma} \cdot s_{l}\right) \alpha_{j} \\
& =\operatorname{wt}\left(\left|\emptyset_{l}, s_{l}\right\rangle\right)+d\left(s_{l}, \dot{\sigma} . s_{l}\right) \delta-\sum_{j=0}^{n-1} M_{j}\left(w+d\left(s_{l}, \dot{\sigma} . s_{l}\right) \delta ; \dot{\sigma} . s_{l}\right) \alpha_{j},
\end{aligned}
$$

whence $w=\operatorname{wt}\left(\left|\emptyset_{l}, s_{l}\right\rangle\right)-\sum_{j=0}^{n-1} M_{j}\left(w+d\left(s_{l}, \dot{\sigma} . s_{l}\right) \delta ; \dot{\sigma} . s_{l}\right) \alpha_{j}$. The lemma follows.

\section{Lemma 4.8 Keep Notation 4.1. Assume that}

$$
s_{i}-s_{i+1} \geq n\left(M_{0}\left(w ; s_{l}\right)+1\right)
$$

(where we put $s_{0}:=n+s_{l}$ if $i=0$ ). Then $\dot{w}+\dot{\alpha}_{i}$ is not a weight of $\mathbf{F}_{p}\left[s_{n}\right]^{\bullet}$.

Proof Assume on the contrary that $\dot{w}+\dot{\alpha}_{i}$ is a weight of $\mathbf{F}_{p}\left[\boldsymbol{s}_{n}\right]^{\bullet}$. Let $\left(\lambda_{l}, \boldsymbol{t}_{l}\right) \in$ $\Pi^{l} \times \mathbb{Z}^{l}(s)$ be such that $\dot{\mathrm{wt}}\left(\left|\lambda_{l}, \boldsymbol{t}_{l}\right\rangle\right)=\dot{w}+\dot{\alpha}_{i}$. Write $\dot{w}=d \dot{\delta}+\sum_{j=1}^{l} b_{j} \dot{\Lambda}_{j-1}$ with $b_{1}, \ldots, b_{l}, d \in \mathbb{Z}$. Then by (2), we have $\dot{w}+\dot{\alpha}_{i}=\left(d+\delta_{i, 0}\right) \dot{\delta}+$ $\sum_{j=1}^{l}\left(b_{j}+a_{i, j-1}\right) \dot{\Lambda}_{j-1}$, where $\left(a_{i^{\prime}, j^{\prime}}\right)_{0 \leq i^{\prime}, j^{\prime} \leq l-1}$ is the Cartan matrix of $\widehat{\mathfrak{s l}}_{l}$. Since the vectors $\dot{\Lambda}_{0}, \ldots, \dot{\Lambda}_{l-1}$ and $\dot{\delta}$ are linearly independent, we get by (33) the following relations:

$$
\begin{aligned}
-\left(\Delta\left(\boldsymbol{t}_{l}, n\right)+M_{0}\left(\lambda_{l} ; \boldsymbol{t}_{l} ; n\right)\right) & =-\left(\Delta\left(s_{l}, n\right)+M_{0}\left(w ; s_{l}\right)\right)+\delta_{i, 0}, \\
\boldsymbol{t}_{l} & =\left\{\begin{array}{cc}
\left(s_{1}, \ldots, s_{i-1}, s_{i}+1, s_{i+1}-1, s_{i+2}, \ldots, s_{l}\right) & \text { if } i \geq 1, \\
\left(s_{1}-1, s_{2}, \ldots, s_{l-1}, s_{l}+1\right) & \text { if } i=0 .
\end{array}\right.
\end{aligned}
$$

For $a \in \mathbb{Z}$, denote temporarily by $\bar{a} \in \llbracket 0 ; n-1 \rrbracket$ the residue of $a$ modulo $n$. Using $(* *)$ and the fact that $s_{0}-\overline{s_{0}}=1+\left(s_{l}-\overline{s_{l}}\right)$, we get

$$
\Delta\left(\boldsymbol{t}_{l}, n\right)=\Delta\left(s_{l}, n\right)+\frac{1}{n}\left(s_{i}-s_{i+1}\right)+\frac{1}{n}\left(\overline{s_{i+1}}-\overline{s_{i}}\right)+\delta_{\overline{s_{i+1}}, 0}-\delta_{i, 0} . \quad(* * *)
$$


Moreover, by assumption we have $M_{0}\left(w ; s_{l}\right)-\frac{1}{n}\left(s_{i}-s_{i+1}\right) \leq-1$. This together with $(*)$ and $(* * *)$ imply

$$
\begin{aligned}
M_{0}\left(\lambda_{l} ; \boldsymbol{t}_{l} ; n\right) & =-\frac{1}{n}\left(\overline{s_{i+1}}-\overline{s_{i}}\right)-\delta_{\overline{s_{i+1}}, 0}+M_{0}\left(w ; s_{l}\right)-\frac{1}{n}\left(s_{i}-s_{i+1}\right) \\
& \leq-\frac{1}{n}\left(\overline{s_{i+1}}-\overline{s_{i}}\right)-\delta_{\overline{s_{i+1}}, 0}-1<0,
\end{aligned}
$$

which is absurd since $M_{0}\left(\lambda_{l} ; \boldsymbol{t}_{l} ; n\right)$ is the number of 0 -nodes of the multi-partition $\lambda_{l}$.

Remark 4.9 The lower bound $s_{i}-s_{i+1} \geq n\left(M_{0}\left(w ; s_{l}\right)+1\right)$ from Lemma 4.8 is certainly not the best to ensure that $\dot{w}+\dot{\alpha}_{i} \notin \dot{\mathcal{P}}\left(\mathbf{F}_{p}\left[s_{n}\right]^{\bullet}\right)$. We actually conjecture that the latter statement holds if

$$
s_{i}-s_{i+1} \geq M_{0}\left(w ; s_{l}\right)+\cdots+M_{n-1}\left(w ; s_{l}\right)
$$

(this lower bound is in general better).

\subsection{A graph containing multi-charges conjugated under the action of $\dot{W}_{l}$}

Definition 4.10 Fix $\boldsymbol{r}_{l} \in A_{l, n}(s)$ and $M \in \mathbb{N}^{*}$. (Recall that the set $A_{l, n}(s)$ defined by (38) is a fundamental domain for the action of $\dot{W}_{l}$.) For $s_{l}=\left(s_{1}, \ldots, s_{l}\right) \in \dot{W}_{l} . \boldsymbol{r}_{l}$, $\boldsymbol{t}_{l} \in \dot{W}_{l} . \boldsymbol{r}_{l}, 0 \leq i \leq l-1$, write $\boldsymbol{s}_{l} \stackrel{i}{\longrightarrow} \boldsymbol{t}_{l}$ if $\boldsymbol{t}_{l}=\dot{\sigma}_{i} . s_{l}$ and $s_{i}-s_{i+1} \geq M$ (if $i=0$, we put $\left.s_{0}:=n+s_{l}\right)$. Let $\Gamma(M)$ be the graph containing $\dot{W}_{l} . r_{l}$ as set of vertices and the arrows $\boldsymbol{s}_{l} \stackrel{i}{\longrightarrow} \boldsymbol{t}_{l}\left(\boldsymbol{s}_{l}, \boldsymbol{t}_{l} \in \dot{W}_{l} . \boldsymbol{r}_{l}, 0 \leq i \leq l-1\right)$.

Remark 4.11 Note that $\Gamma(1)$ is connected. More generally, we claim that $\Gamma(M)$ has finitely many connected components. To see this, introduce the following notation. For $s_{l} \in \dot{W}_{l} . r_{l}$, let $\dot{\sigma}\left(s_{l}\right) \in \dot{W}_{l}$ be the element of minimal length such that $\boldsymbol{s}_{l}=\dot{\sigma}\left(\boldsymbol{s}_{l}\right) . \boldsymbol{r}_{l}$, and let $\ell\left(\boldsymbol{s}_{l}\right)$ denote the length of $\dot{\sigma}\left(\boldsymbol{s}_{l}\right)$. Now, for each connected component $C$ in $\Gamma(M)$, choose $s_{l}(C) \in C$ such that $\ell\left(s_{l}(C)\right)$ is minimal in the set $\left\{\ell\left(\boldsymbol{t}_{l}\right) \mid \boldsymbol{t}_{l} \in C\right\}$. (We think that this determines $\boldsymbol{s}_{l}(C)$ in a unique way, but we do not have the proof for this fact.) One can then show easily that $s_{l}(C)$ lies in the finite set

$$
\left\{\left(t_{1}, \ldots, t_{l}\right) \in \mathbb{Z}^{l}(s) \mid \forall 0 \leq i \leq l-1, t_{i+1}-t_{i} \leq M-1\right\},
$$

which proves the claim.

We now give a relation between (the connected components of) $\Gamma(M)$ and the comparison of canonical bases. We shall give an important application of this result in Section 5.

Proposition 4.12 Let $\boldsymbol{r}_{l} \in A_{l, n}(s)$ and $w \in \mathcal{P}\left(\mathbf{F}_{q}\left[\boldsymbol{r}_{l}\right]\right)$. Put $M:=n\left(M_{0}\left(w ; \boldsymbol{r}_{l}\right)+1\right)$. Let $\boldsymbol{s}_{l}$ and $\boldsymbol{t}_{l}$ be two multi-charges in the same connected component of $\Gamma(M)$ (in particular, $\boldsymbol{s}_{l}$ and $\boldsymbol{t}_{l}$ are in the $\dot{W}_{l}$-orbit of $\boldsymbol{r}_{l}$ ). Then the canonical bases of $\mathbf{F}_{q}\left[\boldsymbol{s}_{l}\right]\left\langle w+d\left(\boldsymbol{r}_{l}, \boldsymbol{s}_{l}\right) \delta\right\rangle$ and $\mathbf{F}_{q}\left[\boldsymbol{t}_{l}\right]\left\langle w+d\left(\boldsymbol{r}_{l}, \boldsymbol{t}_{l}\right) \delta\right\rangle$ are similar in the sense of Definition 3.2. 
Proof We may assume that $\boldsymbol{s}_{l} \stackrel{i}{\longrightarrow} \boldsymbol{t}_{l}$ with $i \in \llbracket 0 ; l-1 \rrbracket$. With obvious notation, we have by Lemma $4.7: s_{i}-s_{i+1} \geq n\left(M_{0}\left(w ; \boldsymbol{r}_{l}\right)+1\right)=n\left(M_{0}\left(w+d\left(\boldsymbol{r}_{l}, \boldsymbol{s}_{l}\right) \delta ; \boldsymbol{s}_{l}\right)+1\right)$. We can therefore apply Lemma 4.8 and then Theorem 4.4 to conclude.

With the notation above, Proposition 4.12 and Remark 4.11 show that there are only finitely many similarity classes of canonical bases of $\mathbf{F}_{q}\left[\boldsymbol{s}_{l}\right]\left\langle w+d\left(\boldsymbol{r}_{l}, \boldsymbol{s}_{l}\right) \delta\right\rangle$, where $s_{l}$ ranges over the $\dot{W}_{l}$-orbit of $\boldsymbol{r}_{l}$ and $\left(\boldsymbol{r}_{l}, w\right)$ is fixed.

\section{Comparison of canonical bases for dominant multi-charges}

Definition 5.1 Let $M \in \mathbb{N}$. We say that $\left(x_{1}, \ldots, x_{N}\right) \in \mathbb{R}^{N}$ is $M$-dominant if for all $1 \leq i \leq N-1$, we have

$$
x_{i}-x_{i+1} \geq M
$$

Throughout Section 5, we keep the following notation.

\subsection{Notation}

* Recall that $\mathbb{R}^{l}(s)=\left\{\left(x_{1}, \ldots, x_{l}\right) \in \mathbb{R}^{l} \mid x_{1}+\cdots+x_{l}=s\right\}$. The subset of $\mathbb{R}^{l}(s)$ formed by the $M$-dominant elements will be denoted by $\mathcal{C}_{M}$.

* The group $\dot{W}_{l} \cong \widetilde{\mathfrak{S}}_{l}$ is a semidirect product of the finite symmetric group $\mathfrak{S}_{l}$ and an Abelian group $\dot{\mathcal{Q}}$ which is free of rank $l-1$. More precisely, $\dot{\mathcal{Q}}$ is spanned by $\dot{\tau}_{1}, \ldots, \dot{\tau}_{l-1}$, where $\dot{\tau}_{i}(1 \leq i \leq l-1)$ acts on $\mathbb{Z}^{l}(s)$ by

$$
\dot{\tau}_{i} .\left(s_{1}, \ldots, s_{l}\right)=\left(s_{1}, \ldots, s_{i-1}, s_{i}+n, s_{i+1}-n, s_{i+2}, \ldots, s_{l}\right) \quad\left(\left(s_{1}, \ldots, s_{l}\right) \in \mathbb{Z}^{l}(s)\right)
$$

(since $\dot{W}_{l}$ acts faithfully on $\mathbb{Z}^{l}(s)$, this determines $\dot{\tau}_{i}$ completely).

* For $\boldsymbol{a}_{l}=\left(a_{1}, \ldots, a_{l}\right) \in \mathbb{Z}^{l}$, put

$$
\mathcal{L}_{\boldsymbol{a}_{l}}:=\left\{\left(s_{1}, \ldots, s_{l}\right) \in \mathbb{Z}^{l}(s) \mid \forall 1 \leq i \leq l, s_{i} \equiv a_{i} \quad(\bmod n)\right\} .
$$

Note that $\dot{\mathcal{Q}}$ acts transitively on $\mathcal{L}_{\boldsymbol{a}_{l}}$; in particular, two elements of $\mathcal{L}_{\boldsymbol{a}_{l}}$ lie in a same $\dot{W}_{l}$-orbit.

* For $\boldsymbol{s}_{l}, \boldsymbol{t}_{l} \in \mathbb{Z}^{l}(s)$ and $M \in \mathbb{N}$, write

$$
\boldsymbol{s}_{l} \underset{\bar{M}}{\equiv} \boldsymbol{t}_{l}
$$

if $\mathcal{L}_{\boldsymbol{s}_{l}}=\mathcal{L}_{\boldsymbol{t}_{l}}$, and there exist $\boldsymbol{s}_{l}^{(0)}, \ldots, \boldsymbol{s}_{l}^{(r)} \in \mathcal{L}_{\boldsymbol{s}_{l}} \cap \mathcal{C}_{M}$ such that $\boldsymbol{s}_{l}^{(0)}=\boldsymbol{s}_{l}, \boldsymbol{s}_{l}^{(r)}=\boldsymbol{t}_{l}$ and for all $1 \leq i \leq r$, we have $s_{l}^{(i)} \in\left\{\dot{\tau}_{j}^{ \pm 1} \cdot s_{l}^{(i-1)} \mid 1 \leq j \leq l-1\right\}$. In other words, put into a non-oriented graph all the elements of $\mathbb{Z}^{l}(s)$ and draw an edge between two vertices if they are $\dot{W}_{l}$-conjugated to each other by a generator of $\dot{\mathcal{Q}}$ or its inverse. Then $s_{l} \underset{M}{\equiv} t_{l}$ if and only if there exists a path in this graph connecting $s_{l}$ and $\boldsymbol{t}_{l}$ through $M$-dominant vertices (including $\boldsymbol{s}_{l}$ and $\boldsymbol{t}_{l}$ ). 
* Let $\boldsymbol{r}_{l}=\left(r_{1}, \ldots, r_{l}\right) \in A_{l, n}(s)$ and $w \in \mathcal{P}\left(\mathbf{F}_{q}\left[\boldsymbol{r}_{l}\right]\right)$. Recall the definition of the integers $d\left(\boldsymbol{r}_{l}, \boldsymbol{s}_{l}\right)\left(\boldsymbol{s}_{l} \in \mathbb{Z}^{l}(s)\right)$ from $(57)$ and $M_{i}\left(w ; \boldsymbol{r}_{l}\right)(0 \leq i \leq n-1)$ from Notation 4.6.

\subsection{A third theorem of comparison}

The goal of Section 5 is to prove the following theorem.

\section{Theorem 5.2}

Keep Notation 5.1. Then there exists $N \in \mathbb{N}$ (which only depends on $n, l$ and $\left.M_{0}\left(w ; \boldsymbol{r}_{l}\right)\right)$ such that for all $N$-dominant multi-charges $\boldsymbol{s}_{l}, \boldsymbol{t}_{l} \in \dot{W}_{l} . \boldsymbol{r}_{l}$ with $\mathcal{L}_{\boldsymbol{s}_{l}}=\mathcal{L}_{\boldsymbol{t}_{l}}$, the canonical bases of $\mathbf{F}_{q}\left[\boldsymbol{s}_{l}\right]\left\langle w+d\left(\boldsymbol{r}_{l}, \boldsymbol{s}_{l}\right) \delta\right\rangle$ and $\mathbf{F}_{q}\left[\boldsymbol{t}_{l}\right]\left\langle w+d\left(\boldsymbol{r}_{l}, \boldsymbol{t}_{l}\right) \delta\right\rangle$ are similar.

Remark 5.3 The proof of Theorem 5.1 will provide an integer $N$ of the form

$$
N=n M_{0}\left(w ; \boldsymbol{r}_{l}\right)+c,
$$

where $c$ is a constant that can be explicitly calculated. This $c$ a priori depends on the multi-charge $\boldsymbol{r}_{l} \in A_{l, n}(s)$ that we have fixed in Notation 5.1. However, by taking a maximum over the finite set $A_{l, n}(s)$, we can make $c$ independent of $\boldsymbol{r}_{l}$ (see the proof of Proposition 5.6). More precisely, by Remark 5.13 we can take $c \geq n\left(l^{2}+l+3\right)$. However, the corresponding value of $N$ is probably not optimal. Indeed, according to Remark 4.9 and explicit calculus of canonical bases, we conjecture that Theorem 5.2 holds if we replace this $N$ by

$$
N^{\prime}:=M_{0}\left(w ; \boldsymbol{r}_{l}\right)+\cdots+M_{n-1}\left(w ; \boldsymbol{r}_{l}\right)
$$

(the latter lower bound is in general better).

Example 5.4 Take $n=3, l=2, \boldsymbol{r}_{l}=(1,0)$ and $w=\operatorname{wt}\left(\left|\emptyset_{l}, \boldsymbol{r}_{l}\right\rangle\right)-\left(\alpha_{0}+\alpha_{1}+\alpha_{2}\right)$. With notation from Theorem 5.2 and Remark 5.3, we can take $N=30$ and $N^{\prime}=3$. Note that all the multi-charges $s_{l}^{(k)}:=(3 k+1,-3 k)(k \in \mathbb{Z})$ are in the $\dot{W}_{l}$-orbit of $\boldsymbol{r}_{l}$ and they have the same pair of residues modulo $n$. Put $w_{k}:=w+d\left(\boldsymbol{r}_{l}, \boldsymbol{s}_{l}^{(k)}\right) \delta$ $(k \in \mathbb{Z})$. By Theorem 5.2, the canonical bases of $\mathbf{F}_{q}\left[\boldsymbol{s}_{l}^{(k)}\right]\left\langle w_{k}\right\rangle, k \geq 5$ are pairwise similar. By Remark 5.3, the canonical bases of $\mathbf{F}_{q}\left[\boldsymbol{s}_{l}^{(k)}\right]\left\langle w_{k}\right\rangle, k \geq 1$ should be pairwise similar, which can be actually checked for $1 \leq k \leq 5$ by explicit calculus. Namely, the transition matrices $\Delta_{k}^{\epsilon}(q)$ of the canonical bases of $\mathbf{F}_{q}\left[s_{l}^{(k)}\right]\left\langle w_{k}\right\rangle(k \geq 1, \epsilon= \pm 1)$ are

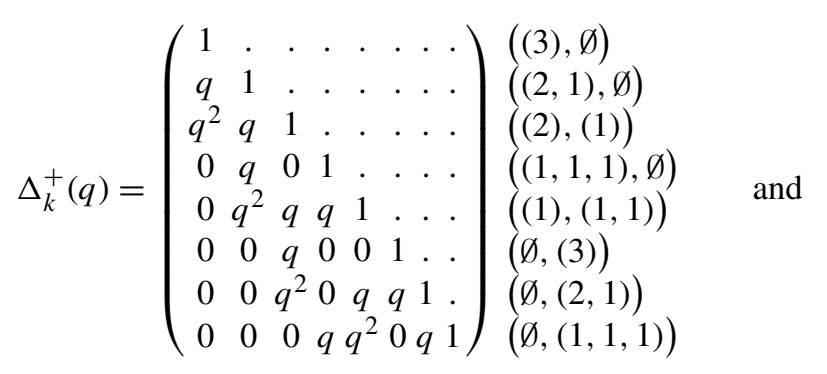




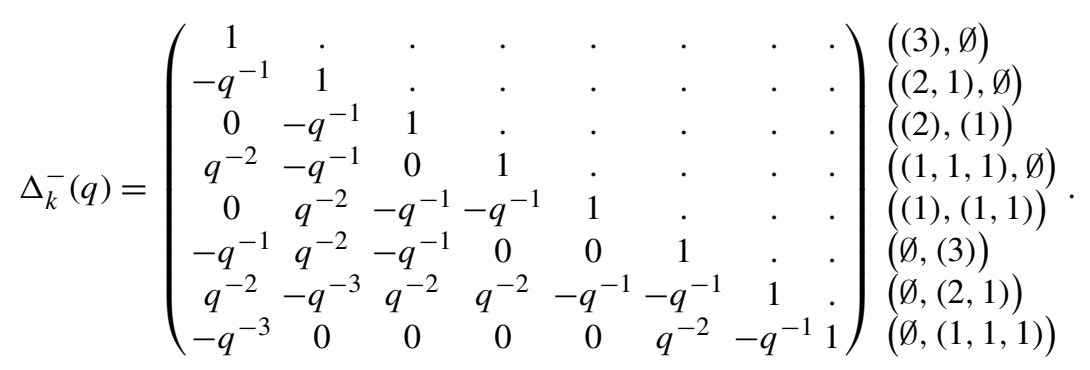

One can check moreover that

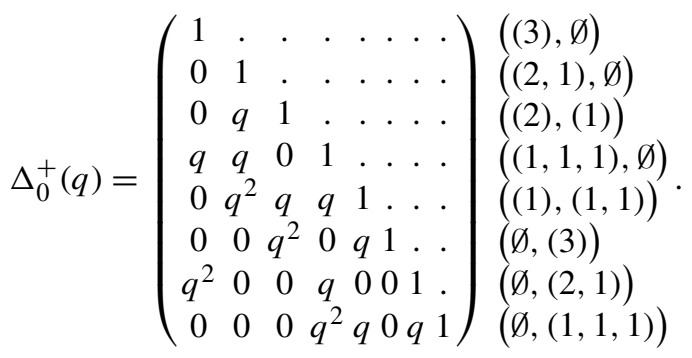

Note that $\Delta_{1}^{+}(q)$ has 22 nonzero entries, whereas $\Delta_{0}^{+}(q)$ has only 21 nonzero entries. As a consequence, the canonical bases of $\mathbf{F}_{q}\left[\boldsymbol{s}_{l}^{(k)}\right]\left\langle w_{k}\right\rangle$ for $k=0$ and $k=1$ are not similar.

The proof of Theorem 5.2 relies on the two following propositions.

Proposition 5.5 Keep Notation 5.1. Let $\boldsymbol{s}_{l} \in \dot{W}_{l} . \boldsymbol{r}_{l}$ be an $M$-dominant multi-charge with $M:=n\left(M_{0}\left(w ; \boldsymbol{r}_{l}\right)+2\right)$. Let $1 \leq i \leq l-1$ and $\boldsymbol{t}_{l}:=\dot{\tau}_{i} . s_{l}$. Then the canonical bases of $\mathbf{F}_{q}\left[\boldsymbol{s}_{l}\right]\left\langle w+d\left(\boldsymbol{r}_{l}, \boldsymbol{s}_{l}\right) \delta\right\rangle$ and $\mathbf{F}_{q}\left[\boldsymbol{t}_{l}\right]\left\langle w+d\left(\boldsymbol{r}_{l}, \boldsymbol{t}_{l}\right) \delta\right\rangle$ are similar.

Proof We give the proof for $2 \leq i \leq l-3$ (the proof for $i=1, i=l-2$ and $i=l-1$ is similar). One easily checks that

$$
\dot{\tau}_{i}=\dot{\sigma}_{i-1} \dot{\sigma}_{i-2} \cdots \dot{\sigma}_{1} \dot{\sigma}_{0} \dot{\sigma}_{l-1} \cdots \dot{\sigma}_{i+2} \dot{\sigma}_{i+1} \dot{\sigma}_{i+2} \cdots \dot{\sigma}_{l-1} \dot{\sigma}_{0} \dot{\sigma}_{1} \cdots \dot{\sigma}_{i}
$$

Let $0 \leq k \leq 2 l-2$. Denote by $\dot{\tau}_{i}[k]$ the right factor of length $k$ in this word (we thus have $\dot{\tau}_{i}[0]=\mathrm{id}, \dot{\tau}_{i}[1]=\dot{\sigma}_{i}, \dot{\tau}_{i}[2]=\dot{\sigma}_{i-1} \dot{\sigma}_{i}$ and so on). Put

$$
\boldsymbol{s}_{l}^{(k)}=\left(s_{1}^{(k)}, \ldots, s_{l}^{(k)}\right):=\dot{\tau}_{i}[k] . s_{l} .
$$

For $1 \leq k \leq 2 l-2$, let $i_{k} \in \llbracket 0 ; l-1 \rrbracket$ be such that $\dot{\tau}_{i}[k]=\dot{\sigma}_{i_{k}} \dot{\tau}_{i}[k-1]$. Let now $0 \leq k \leq 2 l-3$. By computing the action of $\dot{\tau}_{i}[k]$ on $s_{l}$, one can show the following:

(i) $s_{i_{k+1}}^{(k)}, s_{i_{k+1}+1}^{(k)} \in\left\{s_{1}, \ldots, s_{l}, s_{l}+n, s_{i}+n, s_{i+1}-n\right\}$.

(ii) If $s_{i_{k+1}}^{(k)}=s_{a}+\epsilon n$ and $s_{i_{k+1}+1}^{(k)}=s_{a^{\prime}}+\epsilon^{\prime} n$ with $a, a^{\prime} \in \llbracket 1 ; l \rrbracket, \epsilon, \epsilon^{\prime} \in\{-1,0,1\}$, then $a \neq a^{\prime}$ and $\epsilon \epsilon^{\prime}=0$. 
Note moreover that by assumption on $s_{l}$, we have for all $a, b \in \llbracket 1 ; l \rrbracket$ such that $a \neq b$, $\left|s_{b}-s_{a}\right| \geq M|b-a| \geq M$. This together with (i), (ii) imply that

$$
\left|s_{i_{k+1}}^{(k)}-s_{i_{k+1}+1}^{(k)}\right| \geq M-n .
$$

As a consequence, $\Gamma(M-n)$ contains the arrow $\boldsymbol{s}_{l}^{(k)} \stackrel{i_{k}}{\longrightarrow} \boldsymbol{s}_{l}^{(k+1)}$ or $\boldsymbol{s}_{l}^{(k+1)} \stackrel{i_{k}}{\longrightarrow} \boldsymbol{s}_{l}^{(k)}$. It follows that $s_{l}$ and $\boldsymbol{t}_{l}$ are in the same connected component of $\Gamma(M-n)=$ $\Gamma\left(n\left(M_{0}\left(w ; \boldsymbol{r}_{l}\right)+1\right)\right)$. We can therefore apply Proposition 4.12 to conclude.

Proposition 5.6 Keep Notation 5.1. Let $M \in \mathbb{N}$. Then there exists $c \in \mathbb{Z}$ (which only depends on $l$ and $n$, but not on $M$ nor $\left.\boldsymbol{r}_{l}\right)$ such that for all $(M+c)$-dominant multicharges $\boldsymbol{s}_{l}, \boldsymbol{t}_{l} \in \dot{W}_{l} . \boldsymbol{r}_{l}$ with $\mathcal{L}_{\boldsymbol{s}_{l}}=\mathcal{L}_{\boldsymbol{t}_{l}}$, we have $\boldsymbol{s}_{l} \underset{\bar{M}}{\equiv} \boldsymbol{t}_{l}$.

Proof We shall prove this proposition in Section 5.3.

Proof of Theorem 5.2 from Propositions 5.5 and 5.6. Let $M:=n\left(M_{0}\left(w ; \boldsymbol{r}_{l}\right)+2\right)$. Let $c \in \mathbb{Z}$ be the integer given by Proposition 5.6 and put $N:=M+c$. Let $\boldsymbol{s}_{l}, \boldsymbol{t}_{l} \in$ $\dot{W}_{l} . \boldsymbol{r}_{l}$ be two $N$-dominant multi-charges such that $\mathcal{L}_{\boldsymbol{s}_{l}}=\mathcal{L}_{\boldsymbol{t}_{l}}$. Put $\mathcal{L}:=\mathcal{L}_{\boldsymbol{s}_{l}}=\mathcal{L}_{\boldsymbol{t}_{l}}$. By Proposition 5.6, there exist $\boldsymbol{s}_{l}^{(0)}, \ldots, \boldsymbol{s}_{l}^{(r)} \in \mathcal{L} \cap \mathcal{C}_{M}$ such that $\boldsymbol{s}_{l}^{(0)}=\boldsymbol{s}_{l}, \boldsymbol{s}_{l}^{(r)}=\boldsymbol{t}_{l}$ and for all $1 \leq i \leq r$, we have $s_{l}^{(i)} \in\left\{\dot{\tau}_{j}^{ \pm 1} \cdot s_{l}^{(i-1)} \mid 1 \leq j \leq l-1\right\}$. Let $1 \leq i \leq r$. Since $\mathcal{L}_{\boldsymbol{s}_{l}^{(i-1)}}=\mathcal{L}_{\boldsymbol{s}_{l}^{(i)}}=\mathcal{L}$, we have $\boldsymbol{s}_{l}^{(i-1)}, \boldsymbol{s}_{l}^{(i)} \in \dot{W}_{l} . \boldsymbol{r}_{l}$. By Proposition 5.5, the canonical bases of $\mathbf{F}_{q}\left[\boldsymbol{s}_{l}^{(i-1)}\right]\left\langle w+d\left(\boldsymbol{r}_{l}, \boldsymbol{s}_{l}^{(i-1)}\right) \delta\right\rangle$ and $\mathbf{F}_{q}\left[\boldsymbol{s}_{l}^{(i)}\right]\left\langle w+d\left(\boldsymbol{r}_{l}, \boldsymbol{s}_{l}^{(i)}\right) \delta\right\rangle$ are similar. Theorem 5.2 follows.

\subsection{Proof of Proposition 5.6}

The idea of the proof is the following. Let $s_{l}$ and $\boldsymbol{t}_{l}$ be two multi-charges as in Proposition 5.6 and put $\mathcal{L}:=\mathcal{L}_{\boldsymbol{s}_{l}}=\mathcal{L}_{\boldsymbol{t}_{l}}$. First, we introduce a suitable change of coordinates $\varphi$ that maps $\mathcal{L}$ to $\mathbb{Z}^{l-1}$ and such that $\boldsymbol{a}_{l} \underset{M}{\equiv} \boldsymbol{b}_{l}$ if and only if $\varphi\left(\boldsymbol{a}_{l}\right)$ is $\mathbb{Z}$-connected to $\varphi\left(\boldsymbol{b}_{l}\right)$, that is there exists a piecewise affine path connecting $\varphi\left(\boldsymbol{a}_{l}\right)$ to $\varphi\left(\boldsymbol{b}_{l}\right)$ with edges parallel to the axes of coordinates of $\mathbb{Z}^{l-1}$ (see Lemma 5.8). Roughly speaking, the aim is to replace the lattice $\mathcal{L}$ by $\mathbb{Z}^{l-1}$ and the action of $\dot{\mathcal{Q}}$ by the obvious action of $\mathbb{Z}^{l-1}$ by translations. Doing this, we replace the set of $M$-dominance $\mathcal{C}_{M}$ by a certain cone (that is, an intersection of half-spaces), temporarily denoted by $C_{M}$. Note that two arbitrary points in $C_{M} \cap \mathbb{Z}^{l-1}$ are not necessarily $\mathbb{Z}$-connected in $C_{M}$. However, and this is the second step of the proof, we shall construct an integer $c$ such that $C_{M+c} \subset C_{M}$ and any two points in $C_{M+c} \cap \mathbb{Z}^{l-1}$ are $\mathbb{Z}$-connected in $C_{M}$ (see Proposition 5.12).

Notation 5.7 In addition to Notation 5.1, we shall use for the proof the following notation.

* For $\boldsymbol{x}=\left(x_{1}, \ldots, x_{N}\right) \in \mathbb{R}^{N}\left(N \in \mathbb{N}^{*}\right)$, put

$$
\lfloor\boldsymbol{x}\rfloor:=\left(\left\lfloor x_{1}\right\rfloor, \ldots,\left\lfloor x_{N}\right\rfloor\right) \in \mathbb{Z}^{N}
$$

and define $\lceil\boldsymbol{x}\rceil$ in a similar way. 
Let

$$
A:=\left(\begin{array}{ccccc}
2 & -1 & 0 & \ldots & 0 \\
-1 & 2 & -1 & \ddots & \vdots \\
0 & \ddots & \ddots & \ddots & 0 \\
\vdots & \ddots & -1 & 2 & -1 \\
0 & \ldots & 0 & -1 & 2
\end{array}\right)
$$

denote the Cartan matrix of $\mathfrak{s l}_{l}$. In particular, $A$ has $l-1$ rows and $l-1$ columns.

* For $1 \leq j \leq l-1$, let $\epsilon_{j}:=\left(\delta_{i, j}\right)_{1 \leq i \leq l-1}$ be the $j$-th vector of the natural basis of $\mathbb{R}^{l-1}$. Put also $\mathbf{1}:=\epsilon_{1}+\cdots+\epsilon_{l-1}$.

* Define a partial ordering on the set of matrices (or vectors) of a given size with entries in $\mathbb{R}$ by writing $\boldsymbol{x}=\left(x_{i}\right)_{i \in I} \leq \boldsymbol{y}=\left(y_{i}\right)_{i \in I}$ if $x_{i} \leq y_{i}$ for all $i \in I$. By definition, the maximum of $\boldsymbol{x}$ and $\boldsymbol{y}$ is $\max (\boldsymbol{x}, \boldsymbol{y}):=\left(\max \left(x_{i}, y_{i}\right)\right)_{i \in I}$. One may similarly define the maximum of a greater number of matrices (or vectors) of a given size provided the max in the right hand-side above still exists. Now, for $\boldsymbol{b} \in \mathbb{R}^{l-1}$ define the cones

$$
C_{\boldsymbol{b}}:=\left\{\boldsymbol{x} \in \mathbb{R}^{l-1} \mid A . \boldsymbol{x} \geq \boldsymbol{b}\right\} \quad \text { and } \quad C_{\boldsymbol{b}}^{\prime}:=\left\{\boldsymbol{x} \in \mathbb{R}^{l-1} \mid A . \boldsymbol{x} \leq \boldsymbol{b}\right\} .
$$

The unique vector $\omega=\omega(b) \in \mathbb{R}^{l-1}$ such that $A . \omega=b$ is called the vertex of $C_{b}$ (or $C_{b}^{\prime}$ ).

* For $M \in \mathbb{N}$, let $\boldsymbol{b}(M)=\boldsymbol{b}\left(M ; n, l, \boldsymbol{r}_{l}\right)=\left(b_{1}^{(M)}, \ldots, b_{l-1}^{(M)}\right) \in \mathbb{R}^{l-1}$ denote the vector defined by

$$
b_{i}^{(M)}:=\left(M+r_{i+1}-r_{i}\right) / n \quad(1 \leq i \leq l-1) .
$$

* Define a map $\varphi:\left(s_{1}, \ldots, s_{l}\right) \in \mathbb{R}^{l}(s) \mapsto\left(x_{1}, \ldots, x_{l-1}\right)=\varphi\left(s_{1}, \ldots, s_{l}\right) \in \mathbb{R}^{l-1}$ by

$$
x_{i}:=\frac{1}{n} \sum_{j=1}^{i}\left(s_{j}-r_{j}\right) \quad(1 \leq i \leq l-1) .
$$

Conversely, let $\psi:\left(x_{1}, \ldots, x_{l-1}\right) \in \mathbb{R}^{l-1} \mapsto\left(s_{1}, \ldots, s_{l}\right)=\psi\left(x_{1}, \ldots, x_{l-1}\right) \in$ $\mathbb{R}^{l}(s)$ be the map defined by

$$
s_{i}:=n\left(x_{i}-x_{i-1}\right)+r_{i} \quad(1 \leq i \leq l),
$$

where we put $x_{0}=x_{l}:=0$. (Note that $\varphi$ and $\psi$ depend on the multi-charge $\boldsymbol{r}_{l} \in A_{l, n}(s)$ that we have fixed in Notation 5.1.)

Lemma 5.8 Keep Notation 5.7. Then we have the following. 
(i) The maps $\varphi: \mathbb{R}^{l}(s) \rightarrow \mathbb{R}^{l-1}$ and $\psi: \mathbb{R}^{l-1} \rightarrow \mathbb{R}^{l}(s)$ are bijections inverse to each other.

(ii) We have $\psi\left(\mathbb{Z}^{l-1}\right)=\mathcal{L}_{\boldsymbol{r}_{l}}$, and for $1 \leq i \leq l-1,\left(x_{1}, \ldots, x_{l-1}\right) \in \mathbb{Z}^{l-1}$, we have

$$
\psi\left(x_{1}, \ldots, x_{i}+1, \ldots, x_{l-1}\right)=\dot{\tau}_{i} . \psi\left(x_{1}, \ldots, x_{l-1}\right) .
$$

(iii) For $M \in \mathbb{N}$, we have $\varphi\left(\mathcal{C}_{M}\right)=C_{\boldsymbol{b}(M)}$.

Proof The proof of (i) and (ii) is straightforward. With obvious notation, we have the equivalence

$$
s_{i}-s_{i+1} \geq M \Longleftrightarrow-x_{i-1}+2 x_{i}-x_{i+1} \geq\left(M+r_{i+1}-r_{i}\right) / n
$$

Statement (iii) follows.

Definition 5.9 Let $D \subset \mathbb{R}^{l-1}$. We say that $\boldsymbol{x}, \boldsymbol{y} \in D$ are $\mathbb{Z}$-connected in $D$ if there exist vectors $\boldsymbol{x}^{(0)}, \ldots, \boldsymbol{x}^{(N)} \in D \cap \mathbb{Z}^{l-1}$ such that $\boldsymbol{x}^{(0)}=\boldsymbol{x}, \boldsymbol{x}^{(N)}=\boldsymbol{y}$ and for all $0 \leq i \leq N-1$, we have $\boldsymbol{x}^{(i+1)}-\boldsymbol{x}^{(i)} \in\left\{ \pm \epsilon_{j} \mid 1 \leq j \leq l-1\right\}$; in particular, we have $\boldsymbol{x}, \boldsymbol{y} \in \mathbb{Z}^{l-1}$. In this case, write $\boldsymbol{x}_{D} \boldsymbol{y}$.

In order to prove Proposition 5.6, we have to deal with $\mathbb{Z}$-connected points in cones $C_{\boldsymbol{b}}\left(\boldsymbol{b} \in \mathbb{R}^{l-1}\right)$. Note that two points in $C_{\boldsymbol{b}} \cap \mathbb{Z}^{l-1}$ are not necessary $\mathbb{Z}$-connected in $C_{\boldsymbol{b}}$. For example, take $l=3$ (so $l-1=2$ ) and $\boldsymbol{b}=(0,0)$. Then $\boldsymbol{x}:=(1,1)$ and $\boldsymbol{y}:=(0,0)$ are two points in $C_{\boldsymbol{b}}$ which are not $\mathbb{Z}$-connected in $C_{\boldsymbol{b}}$, because none of the points $(0, \pm 1)$ and $( \pm 1,0)$ lies in $C_{\boldsymbol{b}}$. However, given $\boldsymbol{b} \in \mathbb{R}^{l-1}$, one can construct $\boldsymbol{c} \leq \boldsymbol{b}$ such that any two points in $C_{\boldsymbol{b}}$ with integer coordinates are $\mathbb{Z}$-connected in $C_{\boldsymbol{c}}$. This leads to the introduction of the following set. For $\boldsymbol{b} \in \mathbb{R}^{l-1}$, put

$$
\mathcal{A}(\boldsymbol{b}):=\left\{\boldsymbol{c} \in \mathbb{R}^{l-1} \mid \boldsymbol{c} \leq \boldsymbol{b} \text { and } \forall \boldsymbol{x}, \boldsymbol{y} \in C_{\boldsymbol{b}}, \boldsymbol{x}_{\bar{C}_{\boldsymbol{c}}} \boldsymbol{y}\right\} .
$$

Proposition 5.10 Let $\boldsymbol{b}=\left(b_{1}, \ldots, b_{l-1}\right) \in \mathbb{R}^{l-1}$. Then we have the following.

(i) The set $\mathcal{A}(\boldsymbol{b})$ is nonempty.

(ii) For all $\boldsymbol{c} \in \mathcal{A}(\boldsymbol{b})$, we have $\boldsymbol{c}^{\prime} \leq \boldsymbol{c} \Rightarrow \boldsymbol{c}^{\prime} \in \mathcal{A}(\boldsymbol{b})$.

(iii) The map $\boldsymbol{b} \mapsto \mathcal{A}(\boldsymbol{b})$ is increasing.

(iv) For all $\boldsymbol{b}^{\prime} \in \mathbb{R}^{l-1}$ such that $A^{-1} .\left(\boldsymbol{b}-\boldsymbol{b}^{\prime}\right) \in \mathbb{Z}^{l-1}$, we have $\mathcal{A}(\boldsymbol{b})=\mathcal{A}\left(\boldsymbol{b}^{\prime}\right)+(\boldsymbol{b}-$ $\left.\boldsymbol{b}^{\prime}\right)$.

Proof We prove (i) and leave the other statements to the reader. Let $\omega=$ $\left(\omega_{1}, \ldots, \omega_{l-1}\right)$ be the vertex of $C_{\boldsymbol{b}}, \boldsymbol{b}^{\prime}:=\boldsymbol{b}+2.1$ and $\boldsymbol{\omega}^{\prime}$ be the vertex of $C_{\boldsymbol{b}^{\prime}}$. Let $\omega^{\prime \prime}=\left(\omega_{1}^{\prime \prime}, \ldots, \omega_{l-1}^{\prime \prime}\right)$ be equal to $\max \left(\omega^{\prime},\lceil\omega\rceil\right)$ and let $\omega_{0}^{\prime \prime}=\omega_{l}^{\prime \prime}:=0$. Now let $\boldsymbol{c}=\left(c_{1}, \ldots, c_{l-1}\right) \in \mathbb{R}^{l-1}$ be the vector defined by

$$
c_{i}:=-\omega_{i-1}^{\prime \prime}+2 \omega_{i}-\omega_{i+1}^{\prime \prime} \quad(1 \leq i \leq l-1) .
$$

We shall show that $\boldsymbol{c} \in \mathcal{A}(\boldsymbol{b})$. It is well-known that $A^{-1} \geq 0$, whence $\boldsymbol{x} \in C_{\boldsymbol{b}} \Rightarrow \boldsymbol{x} \geq \boldsymbol{\omega}$ and $\boldsymbol{y} \in C_{\boldsymbol{b}^{\prime}}^{\prime} \Rightarrow \boldsymbol{y} \leq \boldsymbol{\omega}^{\prime}$. Since $\boldsymbol{b} \leq \boldsymbol{b}^{\prime}$, we get $\boldsymbol{\omega} \in C_{\boldsymbol{b}^{\prime}}^{\prime}$ and therefore $\boldsymbol{\omega} \leq \boldsymbol{\omega}^{\prime} \leq \boldsymbol{\omega}^{\prime \prime}$. 
Consider the set

$$
\mathbf{P}:=\left\{\boldsymbol{x} \in \mathbb{R}^{l-1} \mid \boldsymbol{\omega} \leq \boldsymbol{x} \leq \boldsymbol{\omega}^{\prime \prime}\right\} .
$$

The argument above shows that $C_{\boldsymbol{b}} \cap C_{\boldsymbol{b}^{\prime}}^{\prime} \subset \mathbf{P}$, and by construction we also have $\lceil\boldsymbol{\omega}\rceil \in \mathbf{P}$. By definition of $\boldsymbol{c}$, we have $\boldsymbol{\omega} \in \mathbf{P} \subset C_{\boldsymbol{c}}$, whence $\boldsymbol{c} \leq \boldsymbol{b}$. Let us now show that any $\boldsymbol{x} \in C_{\boldsymbol{b}} \cap \mathbb{Z}^{l-1}$ is $\mathbb{Z}$-connected to $\lceil\boldsymbol{\omega}\rceil$ in $C_{\boldsymbol{c}}$. Note that for $\boldsymbol{x}=\left(x_{1}, \ldots, x_{l-1}\right) \in$ $C_{\boldsymbol{b}} \cap \mathbb{Z}^{l-1}$, we have $\boldsymbol{x} \geq \boldsymbol{\omega}$ (because $\boldsymbol{x} \in C_{\boldsymbol{b}}$ ) and therefore $\boldsymbol{x} \geq\lceil\boldsymbol{\top}\rceil$. We can thus argue by induction on

$$
N(\boldsymbol{x}):=\sum_{i=1}^{l-1}\left(x_{i}-\left\lceil\omega_{i}\right\rceil\right) \in \mathbb{N} .
$$

If $N(\boldsymbol{x})=0$, then we have $\boldsymbol{x}=\lceil\boldsymbol{\omega}\rceil \in \mathbf{P} \subset C_{\boldsymbol{c}}$ and we are done. Assume now that $\boldsymbol{x} \in C_{\boldsymbol{b}} \cap \mathbb{Z}^{l-1}, N(\boldsymbol{x})>0$, and consider two cases. Assume first that $\boldsymbol{x} \in C_{\boldsymbol{b}^{\prime}}^{\prime}$. Then we have $\boldsymbol{x} \in C_{\boldsymbol{b}} \cap C_{\boldsymbol{b}^{\prime}}^{\prime} \subset \mathbf{P}$; moreover, we have $\lceil\boldsymbol{\omega}\rceil \in \mathbf{P}$, so $\boldsymbol{x}_{\mathbf{P}}\lceil\boldsymbol{\omega}\rceil$ because any two points in $\mathbf{P} \cap \mathbb{Z}^{l-1}$ are $\mathbb{Z}$-connected. Since $\mathbf{P} \subset C_{\boldsymbol{c}}$, we can conclude in this case. Assume now that $\boldsymbol{x} \notin C_{\boldsymbol{b}^{\prime}}^{\prime}$. Let $1 \leq i \leq l-1$ be such that $-x_{i-1}+2 x_{i}-x_{i+1}>b_{i}+2$ (where we put $x_{0}=x_{l}:=0$ ). Consider the vector

$$
\boldsymbol{y}=\left(y_{1}, \ldots, y_{l-1}\right):=\left(x_{1}, \ldots, x_{i-1}, x_{i}-1, x_{i+1}, \ldots, x_{l-1}\right) \in \mathbb{Z}^{l-1}
$$

and put $y_{0}=y_{l}:=0$. Note that for $1 \leq j \leq l-1$, we have

$$
-y_{j-1}+2 y_{j}-y_{j+1} \geq-x_{j-1}+2 x_{j}-x_{j+1}-2 \delta_{i, j} .
$$

Using this fact together with the definition of $i$ and the assumption $\boldsymbol{x} \in C_{\boldsymbol{b}}$, we get $\boldsymbol{y} \in C_{\boldsymbol{b}}$. Moreover, since $N(\boldsymbol{y})=N(\boldsymbol{x})-1$, we have by induction $\boldsymbol{y}_{C_{\boldsymbol{c}}}\lceil\boldsymbol{\omega}\rceil$. Note also that $\boldsymbol{x}_{C_{c}} \boldsymbol{y}$ because $\boldsymbol{c} \leq \boldsymbol{b}$, hence $\boldsymbol{x}_{C_{c}}\lceil\boldsymbol{}\lceil\rceil$.

Keep Notation 5.7. We now construct an integer $c$ such that for all $M \in \mathbb{N}$, we have $\boldsymbol{b}(M) \in \mathcal{A}(\boldsymbol{b}(M+c))$. Let $\boldsymbol{b} \in \mathbb{R}^{l-1}$. By Proposition 5.10, we can define

$$
m_{M}=m_{M}\left(n, l, \boldsymbol{r}_{l}\right):=\max _{\boldsymbol{c}} \min _{1 \leq i \leq l-1}\left(c_{i}\right) \in \mathbb{Z},
$$

where $c=\left(c_{1}, \ldots, c_{l-1}\right)$ ranges over the set $\mathcal{A}\left(\boldsymbol{b}\left(M ; n, l, \boldsymbol{r}_{l}\right)\right) \cap \mathbb{Z}^{l-1}$.

Lemma 5.11 The sequence $\left(m_{M}\right)_{M \in \mathbb{N}}$ defined by (69) is increasing. Moreover, we have $m_{n l M}=l M+m_{0}$ for all $M \in \mathbb{N}$.

Proof The first statement follows from the fact that the maps $M \rightarrow \boldsymbol{b}(M)$ and $\boldsymbol{b} \mapsto$ $\mathcal{A}(\boldsymbol{b})$ are increasing (the latter by Proposition 5.10). Let $M \in \mathbb{N}, \boldsymbol{b}:=\boldsymbol{b}(n l M)$ and $\boldsymbol{b}^{\prime}:=\boldsymbol{b}(0)$. Since $\operatorname{det}(A)=l$, the Cramer formula shows that $A^{-1} .\left(\boldsymbol{b}-\boldsymbol{b}^{\prime}\right) \in \mathbb{Z}^{l-1}$. Applying Proposition 5.10 (iv) to the pair $\left(\boldsymbol{b}, \boldsymbol{b}^{\prime}\right)$ yields $\mathcal{A}(\boldsymbol{b}(n l M))=\mathcal{A}(\boldsymbol{b}(0))+$ $(l M, \ldots, l M)$. The second statement follows.

finite set.

Put $m_{0}^{(\mathrm{min})}:=\min _{\boldsymbol{a}_{l} \in A_{l, n}(s)} m_{0}\left(n, l, \boldsymbol{a}_{l}\right) \in \mathbb{R}$. This is well-defined because $A_{l, n}(s)$ is a Springer 
Proposition 5.12 With the notation above, put

$$
c:=n+\left\lceil-m_{0}^{(\min )} n\right\rceil+n l \in \mathbb{Z} .
$$

Then $c$ does not depend on $\boldsymbol{r}_{l} \in A_{l, n}(s)$ and for all $M \in \mathbb{N}$, we have $\boldsymbol{b}(M) \in \mathcal{A}(\boldsymbol{b}(M+$ $c)$ ).

Proof The first statement is clear. Let $\boldsymbol{c}=\left(c_{1}, \ldots, c_{l-1}\right) \in \mathcal{A}(\boldsymbol{b}(M+c)) \cap \mathbb{Z}^{l-1}$ be such that $\min _{1 \leq i \leq l-1}\left(c_{i}\right)=m_{M+c}$. By proposition 5.10 (ii), it is enough to show that $\boldsymbol{b}(M) \leq \boldsymbol{c}$. We have

$$
M+c \geq n l\left(\frac{M+n-m_{0} n}{n l}+1\right) \geq n l\left\lceil\frac{M+n-m_{0} n}{n l}\right\rceil=n l\left\lceil\frac{\left(\frac{M}{n}+1\right)-m_{0}}{l}\right\rceil .
$$

By Lemma 5.11, we get

$$
m_{M+c} \geq l\left\lceil\frac{\left(\frac{M}{n}+1\right)-m_{0}}{l}\right\rceil+m_{0} \geq l\left(\frac{\left(\frac{M}{n}+1\right)-m_{0}}{l}\right)+m_{0} \geq\left\lceil\frac{M}{n}\right\rceil .
$$

Let $1 \leq i \leq l-1$. Since $\boldsymbol{r}_{l} \in A_{l, n}(s)$, we have $r_{i+1}-r_{i} \leq 0$, whence

$$
c_{i} \geq m_{M+c} \geq\left\lceil\frac{M}{n}\right\rceil \geq \frac{M}{n}+\frac{r_{i+1}-r_{i}}{n}=b_{i}^{(M)} .
$$

The result follows.

Remark 5.13 The author proved in [21, Lemma 4.43] that $m_{0}^{(\min )} \geq-l^{2}$, which gives an explicit upper bound for the smallest $c \in \mathbb{Z}$ satisfying the conclusions of Propositions 5.12 and 5.6.

Proof of Proposition 5.6 Let $c \in \mathbb{Z}$ be the integer defined in Proposition 5.12. Let $\boldsymbol{s}_{l}$, $\boldsymbol{t}_{l} \in \dot{W}_{l} . \boldsymbol{r}_{l} \cap \mathcal{C}_{M+c}$ be such that $\mathcal{L}_{\boldsymbol{s}_{l}}=\mathcal{L}_{\boldsymbol{t}_{l}}$. By Lemma 5.8, we have $\varphi\left(\boldsymbol{s}_{l}\right), \varphi\left(\boldsymbol{t}_{l}\right) \in$ $C_{\boldsymbol{b}(M+c)} \cap \mathbb{Z}^{l-1}$. Proposition 5.12 now implies that $\boldsymbol{b}(M)$ is in $\mathcal{A}(\boldsymbol{b}(M+c))$, whence

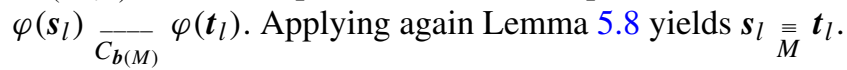

Acknowledgement I thank Bernard Leclerc for his advice and his reading of the preliminary versions of this article.

\section{References}

1. Ariki, S. (1996). On the decomposition numbers of the Hecke algebra of $G(m, 1, n)$. J. Math. Kyoto Univ., 36(4), 789-808.

2. Dipper, R., James, G., \& Mathas, A. (1998). Cyclotomic $q$-Schur algebras. Math. Z., 229, 385-416.

3. Foda, O., Leclerc, B., Okado, M., Thibon, J.-Y., \& Welsh, T. (1999). Branching functions of $A_{n-1}^{(1)}$ and Jantzen-Seitz problem for Ariki-Koike algebras. Adv. Math., 141(2), 322-365.

4. Hayashi, T. (1990). $q$-analogues of Clifford and Weyl algebras-spinor and oscillator representations of quantum enveloping algebras. Commun. Math. Phys., 127(1), 129-144. 
5. Jacon, N. (2004). On the parametrization of the simple modules for Ariki-Koike algebras at roots of unity. J. Math. Kyoto Univ., 44(4), 729-767.

6. Jimbo, M., Misra, K., Miwa, T., \& Okado, M. (1991). Combinatorics of representations of $U_{q}\left(\widehat{\mathfrak{s l}}_{n}\right)$ at $q=0$. Commun. Math. Phys., 136(3), 543-566.

7. Kac, V. G. (1990). Infinite dimensional Lie algebras (3rd edn.). Cambridge: Cambridge University Press.

8. Kashiwara, M. (1993). Global crystal bases of quantum groups. Duke Math. J., 69, 455-485.

9. Kashiwara, M. (1993). The crystal base and Littelmann's refined Demazure character formula. Duke Math. J., 71, 839-958.

10. Kashiwara, M., Miwa, T., \& Stern, E. (1995). Decomposition of $q$-deformed Fock spaces. Sel. Math., $1,787-805$.

11. Kashiwara, M., \& Tanisaki, T. (2002). Parabolic Kazhdan-Lusztig polynomials and Schubert varieties. J. Algebra, 249, 306-325.

12. Lascoux, A., Leclerc, B., \& Thibon, J.-Y. (1996). Hecke algebras at roots of unity and crystal bases of quantum affine algebras. Commun. Math. Phys., 181(1), 205-263.

13. Leclerc, B., \& Miyachi, H. (2002). Some closed formulas for canonical bases of Fock spaces. Represent. Theory, 6, 290-312.

14. Leclerc, B., \& Thibon, J.-Y. (1996). Canonical bases of $q$-deformed Fock spaces. Intern. Math. Res. Not., 9, 447-456.

15. Leclerc, B., \& Thibon, J.-Y. (2000). Littlewood-Richardson coefficients and Kazhdan-Lusztig polynomials. Comb. Methods Represent. Theory Adv. Stud. Pure Math., 28, 155-220.

16. Macdonald, I. G. (1990). Symmetric functions and Hall polynomials (2nd edn.). Oxford Science Publications. London: Oxford University Press.

17. Misra, K. C., \& Miwa, T. (1990). Crystal base for the basic representation of $U_{q}\left(\widehat{\mathfrak{s l}}_{n}\right)$. Commun. Math. Phys., 134(1), 79-88.

18. Scopes, J. (1991). Cartan matrices and Morita equivalence for blocks of the symmetric groups. $J$. Algebra, 142(2), 441-455.

19. Uglov, D. (2000). Canonical bases of higher-level $q$-deformed Fock spaces and Kazhdan-Lusztig polynomials. In M. Kashiwara, T. Miwa (Eds.), Physical combinatorics. Progress in math. (Vol. 191). Birkhäuser, Basel, math.QA/9905196 (1999).

20. Varagnolo, M., \& Vasserot, E. (1999). On the decomposition matrices of the quantized Schur algebra. Duke Math. J., 100, 267-297.

21. Yvonne, X. (2005). Bases canoniques d'espaces de Fock de niveau supérieur. Thèse de l'Université de Caen.

22. Yvonne, X. (2006). A conjecture for $q$-decomposition matrices of cyclotomic $v$-Schur algebras. $J$. Algebra, 304, 419-456.

23. Yvonne, X. (2007). An algorithm for computing the canonical bases of higher-level $q$-deformed Fock spaces. J. Algebra, 309, 760-785. 